\title{
Effective Equations Modeling the Flow of a Viscous Incompressible Fluid through a Long Elastic Tube Arising in the Study of Blood Flow through Small Arteries*
}

\author{
Sunčica Čanićł and Andro Mikelić
}

Abstract. We study the flow of an incompressible viscous fluid through a long tube with compliant walls. The flow is governed by a given time-dependent pressure drop between the inlet and the outlet boundary. The pressure drop is assumed to be small, thereby introducing creeping flow in the tube. Stokes equations for incompressible viscous fluid are used to model the flow, and the equations of a curved, linearly elastic membrane are used to model the wall. Due to the creeping flow and to small displacements, the interface between the fluid and the lateral wall is linearized and supposed to be the initial configuration of the membrane. We study the dynamics of this coupled fluid-structure system in the limit when the ratio between the characteristic width and the characteristic length tends to zero. Using the asymptotic techniques typically used for the study of shells and plates, we obtain a set of Biot-type visco-elastic equations for the effective pressure and the effective displacements. The approximation is rigorously justified through a weak convergence result and through the error estimates for the solution of the effective equations modified by an outlet boundary layer.

Applications of the model problem include blood flow in small arteries. We recover the wellknown law of Laplace and obtain new improved models that hold in cases when the shear modulus of the vessel wall is not negligible and the Poisson ratio is arbitrary.

Key words. fluid-structure interaction, Navier-Stokes equations, asymptotic analysis, blood flow, compliant vessels

AMS subject classifications. 76Z05, 76D07, 35Q30

DOI. $10.1137 / \mathrm{S} 1111111102411286$

1. Introduction. This paper is motivated by the study of blood flow through compliant vessels. One of the open mathematical problems in this research area is rigorous derivation and error analysis of the asymptotic equations that hold in long and narrow axisymmetric vessels. In particular, it is important to analyze fluid-structure interaction resulting from the nonlinear coupling between the incompressible Navier-Stokes equations and the motion of a compliant vessel wall. Modeling of compliant vessel walls is a complex problem in its own right $[11,23]$. Even in the simplified case when the anisotropic behavior of the vessel wall is ignored and angular deformations are neglected, in which case the linear Navier equations for the curved membrane can be used to model the wall [13, 16, 23], the analysis of the nonlinear coupling between the flow equations (Navier-Stokes equations) and wall behavior (Navier membrane equations) is unresolved. See [23] for the closest results. As a first step

\footnotetext{
${ }^{*}$ Received by the editors July 16, 2002; accepted for publication (in revised form) by J. Keener June 17, 2003; published electronically DATE.

http://www.siam.org/journals/siads/x-x/41128.html

${ }^{\dagger}$ Department of Mathematics, University of Houston, 4800 Calhoun Rd., Houston, TX 77204-3476 (canic@math. uh.edu). The research of this author was supported by the Texas Higher Education Board, ARP grant \#0036520112-2001, and by the National Science Foundation under grants DMS-9970310 and DMS-0245513.

${ }^{\ddagger}$ UFR Mathématiques, Université Claude Bernard Lyon 1, Bât. 101, 43, bd. du 11 nov. 1918, 69622 Villeurbanne Cedex, France (amikelic@free.fr).
} 
in analyzing this problem we focus in this paper on understanding the coupling between the Stokes equations (creeping flow) and the Navier equations for a curved elastic membrane. This is a good model for the flow of blood in small arteries. Indeed, it was noted in [20] that in small arteries, viscous effects of blood become more important than the inertia effects, and therefore Stokes equations are appropriate. We study the time-dependent flow governed by a given time-dependent pressure drop between the inlet and the outlet boundary. We refer the reader to books $[5,14,15]$ for a general geometric setting of boundary-value problems in thin domains.

In this paper we derive the reduced (effective) equations that hold for this fluid-structure interaction problem when the ratio $\varepsilon$ between the radius and the length of the vessel is small. The reduced equations are justified by showing that the solution of the original problem converges to the solution of the reduced problem as $\varepsilon \rightarrow 0$. Furthermore, we obtain the precise error estimates which show that the error in the solution of the reduced problem is of order $\mathcal{O}\left(\varepsilon^{2}\right)$ everywhere except at the outlet boundary, where the formation of the boundary layer deteriorates the error to $\mathcal{O}\left(\varepsilon^{3 / 2}\right)$. Derivation of the reduced equations and the precise error estimates are the main original contribution of this work. Numerical simulations showing the dynamics of the fluid-structure interaction and the formation of the boundary layer are presented at the end of the paper.

It is interesting to point out that we recover the well-known law of Laplace [11], namely, the "independent ring model," given by $(5.23)[11,20,4]$, as a special case of the parameter values, namely, in the case when the Poisson ratio of the wall structure is 0.5 and shear modulus of the vessel wall is negligible. For a general Poisson ratio, we provide a general pressure-displacement relationship. Finally, we obtain a model which holds in the situations when the shear modulus is not negligible. Such situations arise, for example, in "stented" arteries (arteries treated with prostheses to prevent occlusion or to prevent aneurysm rupture $[3,10])$. Our analysis provides the reduced equations, which are a more accurate model in these scenarios. Their simple form in terms of the pressure makes the resulting equations particularly attractive for numerical simulations where they can be coupled to the two- or three-dimensional solvers in nonuniform geometries or used, for example, in the study of blood flow in small (e.g., coronary) branching arteries, with the appropriate coupling at the branching locations (see, for example, [21]).

This paper is organized as follows. We define the problem in section 2 . In section 3 we obtain the energy estimates for the above-mentioned fluid-structure interaction problem and show how forcing, given in terms of the pressure drop, controls the elastic and viscous energy. From the energy equality we obtain (optimal) a priori estimates of the solution given in terms of the small parameter $\varepsilon$. The a priori estimates and the choice of the "correct" time-scale (to capture the oscillations induced by the outside forcing and governed by the fluid motion) provide leading order behavior of the state variables and the correct form of the asymptotic expansions. Asymptotic expansions, which are studied on a rescaled domain, are presented in section 4. By inserting the "correct" asymptotic expansions into the coupled fluid-structure system, we obtain the reduced equations in section 5 . The reduced equations are a second-order approximation to the original problem in the interior of the domain. The approximation is rigorously justified by obtaining a weak convergence result and by calculating the corresponding error estimates. The weak convergence result, presented in section 6 , shows 
that the solution of the original problem converges weakly to the unique solution of the asymptotic equations. The error estimates, obtained in section 7 , show the order of the approximation and reveal the formation of a boundary layer at the outlet boundary. See Figure 7.1. The boundary layer at the outlet boundary gives rise to major technical difficulties in the error estimates. To the authors' knowledge, there are no mathematical results on higher order approximations and error estimates for fluid-structure problems in thin domains. This is primarily due to various difficulties associated with dealing with boundary conditions. The closest results on error estimates are the ones presented in [19], where higher order approximations and error estimates for the Stokes flow through fixed domains with small thickness are given, with the given velocity field at the lateral boundary. This is different from our approach. In section 7 we explicitly construct the outlet boundary layer and find the error estimates for the approximate solution modified by the boundary layer solution. We show that due to the presence of the boundary layer, the error in the approximation is of order $\mathcal{O}\left(\varepsilon^{3 / 2}\right)$. This is by $\sqrt{\varepsilon}$ order of magnitude less than the accuracy obtained in [19]. The deterioration of the order of approximation is concentrated at the outlet boundary. This can be seen in the numerical simulations, presented in Figure 7.1.

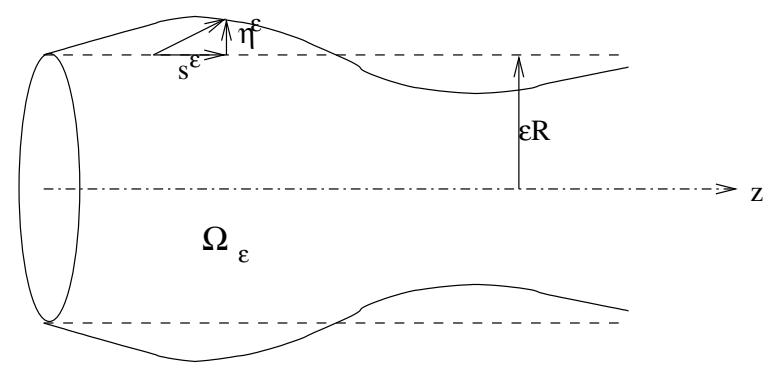

Figure 2.1. Wall displacement.

2. Statement of the problem. We consider the unsteady axisymmetric flow of a Newtonian incompressible fluid in a thin right cylinder whose radius is small with respect to its length. Define the ratio between the radius and the length of the cylinder to be $\epsilon$. For each fixed $\epsilon>0$ introduce $\Omega_{\varepsilon}$ to be

$$
\Omega_{\varepsilon}=\left\{x \in \mathbb{R}^{3} ; x=(r \cos \vartheta, r \sin \vartheta, z), r<\varepsilon R, 0<z<L\right\} .
$$

We assume that the cylinder's lateral wall $\Sigma_{\varepsilon}=\{r=\varepsilon R\} \times(0, L)$ is elastic and that its motion is described in Lagrangian coordinates by the Navier equations

$$
\begin{aligned}
& F_{r}=-\frac{h(\varepsilon) E(\varepsilon)}{1-\sigma^{2}}\left(\frac{\sigma}{\varepsilon R} \frac{\partial s^{\varepsilon}}{\partial z}+\frac{\eta^{\varepsilon}}{\varepsilon^{2} R^{2}}\right)+h(\varepsilon) G(\varepsilon) k(\varepsilon) \frac{\partial^{2} \eta^{\varepsilon}}{\partial z^{2}}-\rho_{w} h(\varepsilon) \frac{\partial^{2} \eta^{\varepsilon}}{\partial t^{2}}, \\
& F_{z}=\frac{h(\varepsilon) E(\varepsilon)}{1-\sigma^{2}}\left(\frac{\partial^{2} s^{\varepsilon}}{\partial z^{2}}+\frac{\sigma}{\varepsilon R} \frac{\partial \eta^{\varepsilon}}{\partial z}\right)-\rho_{w} h(\varepsilon) \frac{\partial^{2} s^{\varepsilon}}{\partial t^{2}} .
\end{aligned}
$$

Here $\eta^{\varepsilon}$ is the radial and $s^{\varepsilon}$ is the longitudinal displacement from the reference state (see Figure 2.1), $h=h(\varepsilon)$ is the membrane thickness, $\rho_{w}$ is the wall volumetric mass, $E=E(\varepsilon)$ is the Young's modulus, $0<\sigma<1 / 2$ is the Poisson ratio, $G=G(\varepsilon)$ is the shear modulus, and 
Table 2.1

Parameter values.

\begin{tabular}{|l|l|}
\hline \hline Parameters & Values \\
\hline \hline$\varepsilon$ & 0.04 \\
\hline Characteristic radius: $\varepsilon R$ & $0.004 \mathrm{~m}$ \\
\hline Dynamic viscosity: $\mu$ & $3.4 \times 10^{-3} \mathrm{~m}^{2} / \mathrm{s}$ \\
\hline Young's modulus: $\mathrm{E}$ & $6000 \mathrm{~Pa},[6]$ \\
\hline Shear modulus: $\mathrm{G}^{*} \mathrm{k}$ & $5 \times 10^{5} \mathrm{~Pa},[11]$ \\
\hline Wall thickness: $\mathrm{h}$ & $4 \times 10^{-4} \mathrm{~m}$ \\
\hline Wall density: $\rho_{w}$ & $1.1 \mathrm{~kg} / \mathrm{m}^{2},[23]$ \\
\hline Blood density: $\rho$ & $1050 \mathrm{~kg} / \mathrm{m}^{3}$ \\
\hline Reference pressure: $P_{0}$ & $13000 \mathrm{~Pa}$ \\
\hline Normalized pressure drop: & $\varepsilon^{1 / 2}$ \\
\hline \hline
\end{tabular}

$k=k(\varepsilon)$ is the Timoshenko shear correction factor (see [16, 23]). $F_{r}$ and $F_{z}$ are the radial and the longitudinal component of the external forces, coming from the stresses induced by the fluid. For the underlying blood-flow problem, the parameter values are presented in Table 2.1. Throughout the paper we will be assuming the following relationships between the parameters in the model.

Assumption 1. The Young's modulus, the wall thickness, and the shear modulus satisfy

$$
\begin{aligned}
h(\varepsilon) E(\varepsilon) & >\varepsilon, \\
\lim _{\varepsilon \rightarrow 0} \frac{h(\varepsilon) E(\varepsilon)}{\varepsilon} & =E_{0} \in(0,+\infty), \\
\lim _{\varepsilon \rightarrow 0} \varepsilon h(\varepsilon) G(\varepsilon) k(\varepsilon) & =G_{0} \in[0,+\infty) .
\end{aligned}
$$

Initially, the cylinder is filled with fluid and the entire structure is in an equilibrium. The equilibrium state has an initial reference pressure $P_{0}$ and the initial velocity is zero. If we denote by $T$ the (membrane) stress tensor, then in the equilibrium (unperturbed) state only the $T_{z z}$ and $T_{\vartheta \vartheta}$ components of the stress tensor corresponding to the curved membrane $\Sigma_{\varepsilon}$ are not zero (see $[16,23]$ ). Their values are $k G$ and $\varepsilon R \Delta P_{0} / h$, respectively, where $\Delta P_{0}$ is the difference between the reference pressure in the tube and the surrounding tissue. For simplicity we assume that $\Delta P_{0}=0$; hence $T_{\vartheta \vartheta}$ is zero in the unperturbed state. A pressure difference between the inlet and the outlet boundary of $\Omega_{\varepsilon}$ creates a deviation from the unperturbed state. We assume that the pressure drop is small compared to the reference pressure and that the fluid acceleration is negligible compared to the effects of the fluid viscosity $\mu$. Therefore, we can use the axially symmetric incompressible Stokes system to model fluid velocity $v^{\varepsilon}=$ $\left(v^{\varepsilon}{ }_{r}, v^{\varepsilon}{ }_{\theta}, v^{\varepsilon} z\right)$ and the pressure perturbation, $p^{\varepsilon}$, from the reference pressure $P_{0}$. Assuming zero angular velocity, in cylindrical coordinates the Eulerian formulation of the problem reads

$$
\begin{aligned}
& -\mu\left(\frac{\partial^{2} v_{r}^{\varepsilon}}{\partial r^{2}}+\frac{\partial^{2} v_{r}^{\varepsilon}}{\partial z^{2}}+\frac{1}{r} \frac{\partial v_{r}^{\varepsilon}}{\partial r}-\frac{v_{r}^{\varepsilon}}{r^{2}}\right)+\frac{\partial p^{\varepsilon}}{\partial r}=0 \text { in } \Omega_{\varepsilon} \times \mathbb{R}_{+}, \\
& -\mu\left(\frac{\partial^{2} v_{z}^{\varepsilon}}{\partial r^{2}}+\frac{\partial^{2} v_{z}^{\varepsilon}}{\partial z^{2}}+\frac{1}{r} \frac{\partial v_{z}^{\varepsilon}}{\partial r}\right)+\frac{\partial p^{\varepsilon}}{\partial z}=0 \text { in } \Omega_{\varepsilon} \times \mathbb{R}_{+}, \\
& \frac{\partial v_{r}^{\varepsilon}}{\partial r}+\frac{\partial v_{z}^{\varepsilon}}{\partial z}+\frac{v_{r}^{\varepsilon}}{r}=0 \quad \text { in } \quad \Omega_{\varepsilon} \times \mathbb{R}_{+} .
\end{aligned}
$$


These equations are coupled with the Navier equations for the curved membrane through the lateral boundary conditions requiring continuity of velocity and continuity of forces at the wall $\Sigma_{\varepsilon}$. More specifically, we require

$$
\begin{array}{llll}
v_{r}^{\varepsilon}=\frac{\partial \eta^{\varepsilon}}{\partial t} & \text { on } & \Sigma_{\varepsilon} \times \mathbb{R}_{+}, \\
v_{z}^{\varepsilon}=\frac{\partial s^{\varepsilon}}{\partial t} & \text { on } & \Sigma_{\varepsilon} \times \mathbb{R}_{+},
\end{array}
$$

and we set the radial and longitudinal forces $F_{r}$ and $F_{z}$ in (2.2) and (2.3) equal to the radial and longitudinal component of the stress exerted by the fluid to the membrane

$$
\begin{array}{llll}
-F_{r}=\left(p^{\varepsilon} I-2 \mu D\left(v^{\varepsilon}\right)\right) \vec{e}_{r} \cdot \vec{e}_{r} & \text { on } & \Sigma_{\varepsilon} \times \mathbb{R}_{+}, \\
-F_{z}=\left(p^{\varepsilon} I-2 \mu D\left(v^{\varepsilon}\right)\right) \vec{e}_{r} \cdot \vec{e}_{z} & \text { on } & \Sigma_{\varepsilon} \times \mathbb{R}_{+},
\end{array}
$$

where $D\left(v^{\varepsilon}\right)$ is the rate of the strain tensor, i.e., the symmetrized gradient of the velocity

$$
D\left(v^{\varepsilon}\right)=\frac{1}{2}\left(\nabla v^{\varepsilon}+\left(\nabla v^{\varepsilon}\right)^{t}\right) .
$$

We note that in this approximation the interface is identified with the reference elastic wall $\Sigma_{\epsilon}$.

The initial state of the structure is unperturbed and at initial velocity zero

$$
\eta^{\varepsilon}=s^{\varepsilon}=\frac{\partial \eta^{\varepsilon}}{\partial t}=\frac{\partial s^{\varepsilon}}{\partial t}=0 \quad \text { on } \quad \Sigma_{\varepsilon} \times\{0\}
$$

and we consider the following boundary data, which, as we shall see in section 6.1, give rise to a well-posed initial-boundary-value problem for the limiting configuration $(\epsilon \rightarrow 0)$ :

$$
\begin{array}{r}
v_{r}^{\varepsilon}=0 \text { and } p^{\varepsilon}=0 \quad \text { on } \quad\left(\partial \Omega_{\varepsilon} \cap\{z=0\}\right) \times \mathbb{R}_{+}, \\
v_{r}^{\varepsilon}=0 \text { and } p^{\varepsilon}=A(t) \quad \text { on } \quad\left(\partial \Omega_{\varepsilon} \cap\{z=L\}\right) \times \mathbb{R}_{+}, \\
\frac{\partial s^{\varepsilon}}{\partial z}=\eta^{\varepsilon}=0 \text { for } z=0, \quad s^{\varepsilon}=\eta^{\varepsilon}=0 \text { for } z=L \text { and } \forall t \in \mathbb{R}_{+} .
\end{array}
$$

Notice that pressure drop $A(t)$ drives the problem. For simplicity we suppose that $A \in$ $C_{0}^{\infty}(0,+\infty)$. Note that physically one should expect nonzero displacements at the outlet boundary. The fixed outlet boundary, required in (2.17), gives rise to the formation of a boundary layer. See sections 6.1 and 7. Periodic boundary conditions, although natural in rigid-wall geometries, do not give rise to well-posed limiting problems when compliant walls are considered.

We summarize the initial-boundary-value problem for the coupled fluid-structure interaction driven by the time-dependent pressure drop between the inlet and the outlet boundary.

Problem $\mathrm{P}^{\varepsilon}$. For each fixed $\varepsilon>0$, find a solution to (2.7), (2.8), and (2.9) in domain $\Omega_{\varepsilon}$ defined by (2.1), with an elastic lateral boundary $\Sigma_{\varepsilon}$. The lateral boundary conditions are given by the continuity of the velocity (2.10) and (2.11) and by the continuity of forces (2.2) and (2.3), where the left-hand sides of (2.2) and (2.3) are substituted by (2.12) and (2.13), 
respectively. The boundary conditions at the inlet and outlet boundaries are (2.15) and (2.16), and the behavior of the elastic wall there is prescribed by (2.17). The initial data is given by (2.14).

In the next section we derive the weak formulation and the energy equality and obtain the a priori estimates for the solution. The existence of a unique weak solution to this problem is straightforward.

\section{Weak formulation and energy estimates.}

3.1. Weak formulation. We define the space of test functions $V^{\varepsilon}$ and the solution space $\mathcal{V}^{\varepsilon}$ to be the following.

Definition 1. The space $V^{\varepsilon} \subset H^{1}\left(\Omega_{\varepsilon}\right)^{3}$ consists of all the axially symmetric functions $\varphi$ such that $\left.\varphi_{r}\right|_{\Sigma_{\varepsilon}},\left.\varphi_{z}\right|_{\Sigma_{\varepsilon}} \in H^{1}(0, L), \varphi_{r}(0, r)=\varphi_{z}(L, \varepsilon R)=\varphi_{r}(L, r)=0$ for $r \leq \varepsilon R$, and $\operatorname{div} \varphi=0$ in $\Omega_{\varepsilon}$.

Definition 2. The space $\mathcal{V}^{\varepsilon}$ consists of all the functions $\left.\left(w_{r}, w_{z}, d_{r}, d_{z}\right) \in H^{1}(0, T) ; V^{\varepsilon}\right) \times$ $\left(H^{1}((0, L) \times(0, T))^{2} \cap H^{2}\left(0, T ; L^{2}(0, L)\right)^{2}\right)$ such that

1. $\frac{\partial w_{r}}{\partial r}+\frac{\partial w_{z}}{\partial z}+\frac{w_{r}}{r}=0$ in $\Omega_{\varepsilon} \times \mathbb{R}_{+}$,

2. $r^{-1} w_{r} \in L^{2}\left((0, T) \times \Omega_{\varepsilon}\right)$,

3. $d_{r}(t, 0)=d_{z}(t, L)=d_{r}(t, L)=0$ on $\mathbb{R}_{+}$,

4. $w_{r}=0$ on $\left(\partial \Omega_{\varepsilon} \cap\{z=0\}\right) \times \mathbb{R}_{+}$, and

5. $w_{r}=\frac{\partial d_{r}}{\partial t}$, and $w_{z}=\frac{\partial d_{z}}{\partial t}$ on $\Sigma_{\varepsilon} \times \mathbb{R}_{+}$.

Recall that for an axially symmetric vector valued function $\psi=\psi_{r} \vec{e}_{r}+\psi_{z} \vec{e}_{z}$ we have

$$
D(\psi)=\left(\begin{array}{ccc}
\frac{\partial \psi_{r}}{\partial r} & 0 & \frac{1}{2}\left(\frac{\partial \psi_{r}}{\partial z}+\frac{\partial \psi_{z}}{\partial r}\right) \\
0 & \frac{\psi_{r}}{r} & 0 \\
\frac{1}{2}\left(\frac{\partial \psi_{r}}{\partial z}+\frac{\partial \psi_{z}}{\partial r}\right) & 0 & \frac{\partial \psi_{z}}{\partial z}
\end{array}\right)
$$

Define the matrix norm $|\cdot|$ through the scalar product

$$
\Xi: \Psi=T_{r}\left(\Xi \cdot \Psi^{t}\right), \Xi, \Psi \in \mathbb{R}^{9} .
$$

Then for each fixed $\varepsilon>0$ the variational formulation and weak solution are defined by the following.

Definition 3. Vector function $\left(v_{r}^{\varepsilon}, v_{z}^{\varepsilon}, \eta^{\varepsilon}, s^{\varepsilon}\right) \in \mathcal{V}^{\varepsilon}$ is a weak solution of problem $\mathrm{P}^{\varepsilon}$ if the following variational formulation is satisfied:

$$
\begin{aligned}
& 2 \mu \int_{\Omega_{\varepsilon}} D\left(v^{\varepsilon}\right): D(\varphi) r d r d z \\
+ & \varepsilon R \int_{0}^{L}\left\{h(\varepsilon) G(\varepsilon) k(\varepsilon) \frac{\partial \eta^{\varepsilon}}{\partial z} \frac{\partial \varphi_{r}}{\partial z}+\frac{h(\varepsilon) E(\varepsilon)}{1-\sigma^{2}}\left(\frac{\sigma}{\varepsilon R} \frac{\partial s^{\varepsilon}}{\partial z}+\frac{\eta^{\varepsilon}}{\varepsilon^{2} R^{2}}\right) \varphi_{r}\right. \\
+ & \left.\frac{h(\varepsilon) E(\varepsilon)}{1-\sigma^{2}}\left(\frac{\partial s^{\varepsilon}}{\partial z} \frac{\partial \varphi_{z}}{\partial z}-\frac{\sigma}{\varepsilon R} \frac{\partial \eta^{\varepsilon}}{\partial z} \varphi_{z}\right)\right\}\left.\right|_{r=\varepsilon R} d z+\left.\varepsilon R \rho_{w} h \frac{d^{2}}{d t^{2}} \int_{0}^{L}\left(\eta^{\varepsilon} \varphi_{r}+s^{\varepsilon} \varphi_{z}\right)\right|_{r=\varepsilon R} d z \\
= & -\left.\int_{0}^{\varepsilon R} A(t) \varphi_{z}\right|_{z=L} r d r \quad \text { in } \quad \mathcal{D}^{\prime}\left(\mathbb{R}_{+}\right) \quad \forall \varphi=\varphi_{r} \vec{e}_{r}+\varphi_{z} \vec{e}_{z} \in V^{\varepsilon}
\end{aligned}
$$


and

$$
\eta^{\varepsilon}=s^{\varepsilon}=\frac{\partial \eta^{\varepsilon}}{\partial t}=\frac{\partial s^{\varepsilon}}{\partial t}=0 \quad \text { on } \quad \Sigma_{\varepsilon} \times\{0\} .
$$

Existence of a unique solution to problem (3.1)-(3.2) is classical. It is a consequence of the corresponding energy estimate and of Korn's inequality [9].

Our goal is to study the behavior of the solution to (3.1)-(3.2) in the limit, as $\varepsilon \rightarrow$ 0 . In order to do that we need an optimal energy estimate with respect to $\varepsilon$. Once the optimal energy estimate is obtained, a priori solution estimates will follow, and the correct asymptotic expansions will be obtained. By plugging the asymptotic expansions into the original equations, the reduced equations, second-order accurate in $\varepsilon$, will be derived. We will see in section 3.2 that obtaining the optimal energy estimate is nonstandard because we do not have the no-slip boundary condition at the lateral boundary and because we will have to deal with the viscous energy which is given in terms of the symmetrized gradient of the velocity $D\left(v^{\varepsilon}\right)$ instead of $\nabla v^{\varepsilon}$.

3.2. Energy estimate. The energy of this problem, obtained by using the velocity field as a test function in (3.1), consists of the elastic energy of the membrane, the viscous energy of the fluid, and the energy due to the outside forcing. The time derivative of the elastic energy

$$
\begin{aligned}
\frac{d \mathcal{E}_{e l}}{d t} & \equiv \varepsilon R \int_{0}^{L}\left\{h(\varepsilon) G(\varepsilon) k(\varepsilon) \frac{\partial \eta^{\varepsilon}}{\partial z} \frac{\partial^{2} \eta^{\varepsilon}}{\partial z \partial t}+\frac{h(\varepsilon) E(\varepsilon)}{1-\sigma^{2}}\left(\left(\frac{\sigma}{\varepsilon R} \frac{\partial s^{\varepsilon}}{\partial z}+\frac{\eta^{\varepsilon}}{\varepsilon^{2} R^{2}}\right) \frac{\partial \eta^{\varepsilon}}{\partial t}\right.\right. \\
& \left.\left.+\left(\frac{\partial s^{\varepsilon}}{\partial z} \frac{\partial^{2} s^{\varepsilon}}{\partial z \partial t}-\frac{\sigma}{\varepsilon R} \frac{\partial \eta^{\varepsilon}}{\partial z} \frac{\partial s^{\varepsilon}}{\partial t}\right)\right)+\varepsilon R \rho_{w} h(\varepsilon)\left(\frac{\partial^{2} \eta^{\varepsilon}}{\partial t^{2}} \frac{\partial \eta^{\varepsilon}}{\partial t}+\frac{\partial^{2} s^{\varepsilon}}{\partial t^{2}} \frac{\partial s^{\varepsilon}}{\partial t}\right)\right\} d z
\end{aligned}
$$

can be expressed as follows.

Lemma 3.1. The displacements $\eta^{\varepsilon}$ and $s^{\varepsilon}$ satisfy

$$
\begin{aligned}
\frac{d \mathcal{E}_{e l}}{d t} & =\varepsilon R \frac{d}{2 d t}\left\{\rho_{w} h(\varepsilon) \int_{0}^{L}\left(\left|\frac{\partial \eta^{\varepsilon}}{\partial t}\right|^{2}+\left|\frac{\partial s^{\varepsilon}}{\partial t}\right|^{2}\right) d z+h(\varepsilon) G(\varepsilon) k(\varepsilon) \int_{0}^{L}\left|\frac{\partial \eta^{\varepsilon}}{\partial z}\right|^{2} d z\right. \\
& \left.+\frac{h(\varepsilon) E(\varepsilon)}{1-\sigma^{2}}\left(\sigma \int_{0}^{L}\left(\frac{\eta^{\varepsilon}}{\varepsilon R}-\frac{\partial s^{\varepsilon}}{\partial z}\right)^{2}+(1-\sigma) \int_{0}^{L}\left(\left|\frac{\eta^{\varepsilon}}{\varepsilon R}\right|^{2}+\left|\frac{\partial s^{\varepsilon}}{\partial z}\right|^{2}\right)\right)\right\} .
\end{aligned}
$$

We are interested in the oscillations of the membrane that are due to the time-dependent pressure drop $A(t)$. These occur at a different time-scale than the characteristic "physical" time. In particular, as we will see later in the text, fluid velocity is greater than the velocity of the displacement. This, in turn, gives rise to long-wavelength elastic waves. It is these waves, among other things, that we would like to keep in our asymptotic reduction. This is why we introduce a new time-scale

$$
\tilde{t}=\omega^{\varepsilon} t
$$

where the characteristic frequency $\omega^{\varepsilon}$ will be specified later (see (3.11)) to include both the waves that occur at the leading order time-scale as well as the oscillations of the membrane caused by a response of the elastic material. The pressure drop is supposed to be a function of $\tilde{t}$. 
From now on we use the rescaled time $\tilde{t}$ and drop the wiggle. By keeping the rescaled time in mind and by using the expression for the elastic energy (3.3), we obtain the following.

Proposition 3.2. Solution $\left(v_{r}^{\varepsilon}, v_{z}^{\varepsilon}, \eta^{\varepsilon}, s^{\varepsilon}\right)$ of problem (3.1)-(3.2) satisfies the variational equality

$$
\begin{aligned}
& \omega^{\varepsilon} h(\varepsilon) \frac{d}{2 d t}\left\{\left(\omega^{\varepsilon}\right)^{2} \rho_{w} \varepsilon R\left(\left\|\frac{\partial \eta^{\varepsilon}(t)}{\partial t}\right\|_{L^{2}(0, L)}^{2}+\left\|\frac{\partial s^{\varepsilon}(t)}{\partial t}\right\|_{L^{2}(0, L)}^{2}\right)+G(\varepsilon) k(\varepsilon) \varepsilon R\left\|\frac{\partial \eta^{\varepsilon}(t)}{\partial z}\right\|_{L^{2}(0, L)}^{2}\right. \\
& \left.+\frac{E(\varepsilon) \varepsilon R}{1-\sigma^{2}}\left(\sigma\left\|\frac{\eta^{\varepsilon}(t)}{\varepsilon R}-\frac{\partial s^{\varepsilon}(t)}{\partial z}\right\|_{L^{2}(0, L)}^{2}+(1-\sigma)\left(\left\|\frac{\eta^{\varepsilon}(t)}{\varepsilon R}\right\|_{L^{2}(0, L)}^{2}+\left\|\frac{\partial s^{\varepsilon}(t)}{\partial z}\right\|_{L^{2}(0, L)}^{2}\right)\right)\right\} \\
& +2 \mu\left\|D\left(v^{\varepsilon}(t)\right)\right\|_{L^{2}\left(\Omega_{\varepsilon}\right)}^{2}=-\int_{0}^{\varepsilon R} A(t) v_{z}^{\varepsilon}(t, r, L) r d r,
\end{aligned}
$$

with $v_{r}^{\varepsilon}=\omega^{\varepsilon} \frac{\partial \eta^{\varepsilon}}{\partial t}$ and $v_{z}^{\varepsilon}=\omega^{\varepsilon} \frac{\partial s^{\varepsilon}}{\partial t}$ on $\Sigma_{\varepsilon} \times(0, T)$.

We now investigate how the energy of the forcing term controls the elastic and the viscous energy of the coupled fluid-structure interaction. We start by transforming and estimating the right-hand side. Since we do not have the no-slip condition for the velocity at the lateral boundary, the situation is more complicated than in the derivation of Reynolds' equation. See [7] and the subsequent papers on the same subject. Furthermore, since on the left-hand side we have only the $L^{2}$-norm of $D\left(v^{\varepsilon}\right)$ and not the $L^{2}$-norm of $\nabla v^{\varepsilon}$, a standard approach based on using the Gronwall estimate and the $L^{2}$-norm of the velocity, $\rho \int_{\Omega_{\varepsilon}}\left|v^{\varepsilon}(t)\right|^{2} r d r d z$, is insufficient to guarantee the correct order of magnitude of the velocity. To get around this difficulty we transform the right-hand side term in (3.5) to a combination of a volume term and a lateral boundary term

$$
-\int_{0}^{\varepsilon R} A(t) v_{z}^{\varepsilon}(t, r, L) r d r=-\int_{\Omega_{\varepsilon}} \frac{A(t)}{L} v_{z}^{\varepsilon} r d r d z+\varepsilon R \int_{0}^{L} A(t) \frac{z}{L} v_{r}^{\varepsilon}(t, \varepsilon R, z) d z
$$

and use the following variant of Biot law which will relate the forcing term with the volume shear stress term in the viscous energy and the elastic energy of the membrane.

Lemma 3.3. The following estimate holds:

$$
\left|\int_{\Omega_{\varepsilon}} v_{z}^{\varepsilon} r d r d z-\omega^{\varepsilon} \frac{\varepsilon^{2} R^{2}}{2} \frac{\partial}{\partial t} \int_{0}^{L} s^{\varepsilon}(t, z) d z\right| \leq \frac{R^{2} \sqrt{L}}{2} \varepsilon^{2}\left\|\frac{1}{2}\left(\frac{\partial v_{z}^{\varepsilon}}{\partial r}+\frac{\partial v_{r}^{\varepsilon}}{\partial z}\right)\right\|_{L^{2}\left(\Omega_{\varepsilon}\right)} .
$$

Proof. The estimate follows by noticing that

$$
\begin{aligned}
\int_{\Omega_{\varepsilon}} v_{z}^{\varepsilon} r d r d z & =\int_{0}^{L}\left[\frac{r^{2}}{2} v_{z}^{\varepsilon}\right]_{0}^{\varepsilon R} d z-\int_{0}^{L} \int_{0}^{\varepsilon R} \frac{r^{2}}{2} \frac{\partial v_{z}^{\varepsilon}}{\partial r} d r d z \\
& =\omega^{\varepsilon} \frac{\partial}{\partial t} \frac{\varepsilon^{2} R^{2}}{2} \int_{0}^{L} s^{\varepsilon}(t, z) d z-\int_{0}^{L} \int_{0}^{\varepsilon R} \frac{r}{2}\left(\frac{\partial v_{z}^{\varepsilon}}{\partial r}+\frac{\partial v_{r}^{\varepsilon}}{\partial z}\right) r d r d z .
\end{aligned}
$$

Throughout the text we will be using the following notation:

$$
\|A(t)\|_{\mathcal{H}}^{2}=\max _{0 \leq \tau \leq t}|A(\tau)|^{2}+\int_{0}^{t}\left|\partial_{\tau} A(\tau)\right|^{2} d \tau
$$


An estimate for the axial component of the displacement in the energy equality (3.5) is given by the following lemma

Lemma 3.4. Axial displacement $s^{\varepsilon}$ satisfies the estimate

$$
\begin{aligned}
& \frac{h(\varepsilon) E(\varepsilon) \varepsilon R}{2(1+\sigma)}\left\|\frac{\partial s^{\varepsilon}}{\partial z}(t)\right\|_{L^{2}(0, L)}^{2}+\frac{\varepsilon^{2} R^{2}}{2 L}\left(A(t) \int_{0}^{L} s^{\varepsilon}(t, z) d z-\int_{0}^{t} \frac{\partial A(\tau)}{\partial \tau} \int_{0}^{L} s^{\varepsilon}(\tau, z) d z d \tau\right) \\
& \geq \frac{h(\varepsilon) E(\varepsilon) \varepsilon R}{4(1+\sigma)}\left\{\left\|\frac{\partial s^{\varepsilon}}{\partial z}(t)\right\|_{L^{2}(0, L)}^{2}+\left(\left\|\frac{\partial s^{\varepsilon}}{\partial z}(t)\right\|_{L^{2}(0, L)}-\frac{R(1+\sigma) \sqrt{L}}{3} \frac{A(t) \varepsilon}{h(\varepsilon) E(\varepsilon)}\right)^{2}\right. \\
& \left.-\int_{0}^{t}\left\|\frac{\partial s^{\varepsilon}}{\partial z}(\tau)\right\|_{L^{2}(0, L)}^{2} d \tau\right\}-\frac{\varepsilon^{3}}{h(\varepsilon) E(\varepsilon)} \frac{(1+\sigma) L R^{3}}{6}\|A(t)\|_{\mathcal{H}}^{2} .
\end{aligned}
$$

Next, an estimate for the radial displacement is given by the following lemma. Lemma 3.5. Radial displacement $\eta^{\varepsilon}$ satisfies the estimate

$$
\begin{aligned}
& \frac{h(\varepsilon) E(\varepsilon)}{2 \varepsilon R(1+\sigma)}\left\|\eta^{\varepsilon}(t)\right\|_{L^{2}(0, L)}^{2}-\frac{\varepsilon R}{L}\left(A(t) \int_{0}^{L} z \eta^{\varepsilon}(t, z) d z-\int_{0}^{t} \frac{\partial A(\tau)}{\partial \tau} \int_{0}^{L} z \eta^{\varepsilon}(\tau, z) d z d \tau\right) \\
& \geq \frac{h(\varepsilon) E(\varepsilon)}{4 \varepsilon R(1+\sigma)}\left\{\left\|\eta^{\varepsilon}(t)\right\|_{L^{2}(0, L)}^{2}-\int_{0}^{t}\left\|\eta^{\varepsilon}(\tau)\right\|_{L^{2}(0, L)}^{2} d \tau\right\}-\frac{\varepsilon^{3}}{h(\varepsilon) E(\varepsilon)} \frac{(1+\sigma) L R^{3}}{3}\|A(t)\|_{\mathcal{H}}^{2} .
\end{aligned}
$$

Combining these two estimates, Lemma 3.3 and the following estimate, which relates the viscous energy with the forcing term,

$$
\varepsilon^{2} \sqrt{\frac{R^{4}}{4 L}}|A(t)|\left\|\frac{1}{2}\left(\frac{\partial v_{z}^{\varepsilon}}{\partial r}+\frac{\partial v_{r}^{\varepsilon}}{\partial z}\right)\right\|_{L^{2}\left(\Omega_{\varepsilon}\right)} \leq \mu\left\|\frac{1}{2}\left(\frac{\partial v_{z}^{\varepsilon}}{\partial r}+\frac{\partial v_{r}^{\varepsilon}}{\partial z}\right)\right\|_{L^{2}\left(\Omega_{\varepsilon}\right)}^{2}+\frac{R^{4} \varepsilon^{4}}{16 \mu L}|A(t)|^{2},
$$

from (3.5) we obtain the following lemma.

Lemma 3.6. The radial displacement $\eta^{\varepsilon}$, the axial displacement $s^{\varepsilon}$, the viscous energy $\mu\left\|D\left(v^{\varepsilon}\right)\right\|_{L^{2}\left(\Omega_{\varepsilon}\right)}^{2}$, and the energy induced by the pressure drop $A(t)$ satisfy the energy estimate

$$
\begin{aligned}
& \omega^{\varepsilon} \frac{h(\varepsilon) E(\varepsilon)}{4 \varepsilon R(1+\sigma)}\left\{\left\|\eta^{\varepsilon}(t)\right\|_{L^{2}(0, L)}^{2}+\varepsilon^{2} R^{2}\left\|\frac{\partial s^{\varepsilon}}{\partial z}(t)\right\|_{L^{2}(0, L)}^{2}\right\}+\mu \int_{0}^{t}\left\|D\left(v^{\varepsilon}\right)(\tau)\right\|_{L^{2}\left(\Omega_{\varepsilon}\right)}^{2} d \tau \\
& \leq \omega^{\varepsilon} \frac{h(\varepsilon) E(\varepsilon)}{4 \varepsilon R(1+\sigma)} \int_{0}^{t}\left\{\left\|\eta^{\varepsilon}(\tau)\right\|_{L^{2}(0, L)}^{2}+\varepsilon^{2} R^{2}\left\|\frac{\partial s^{\varepsilon}}{\partial z}(\tau)\right\|_{L^{2}(0, L)}^{2}\right\} d \tau \\
& +\frac{R^{4} \varepsilon^{4}}{16 \mu L} \int_{0}^{t}|A(\tau)|^{2} d \tau+\omega^{\varepsilon} \frac{\varepsilon^{3}}{h(\varepsilon) E(\varepsilon)} \frac{(1+\sigma) L R^{3}}{2}\|A(t)\|_{\mathcal{H}}^{2} .
\end{aligned}
$$

By applying the Gronwall inequality to (3.9), we get an estimate which is crucial in determining the leading order behavior in asymptotic expansions. The estimate is a basis for the a priori solution estimates in terms of the small parameter $\epsilon$ and the characteristic frequency $\omega^{\varepsilon}$. 
Proposition 3.7. Solution $\left(v_{r}^{\varepsilon}, v_{z}^{\varepsilon}, \eta^{\varepsilon}, s^{\varepsilon}\right)$ of problem (3.1)-(3.2) satisfies the estimate

$$
\begin{gathered}
\omega^{\varepsilon} \frac{h(\varepsilon) E(\varepsilon)}{4 \varepsilon R(1+\sigma)}\left\{\left\|\eta^{\varepsilon}(t)\right\|_{L^{2}(0, L)}^{2}+\varepsilon^{2} R^{2}\left\|\frac{\partial s^{\varepsilon}}{\partial z}(t)\right\|_{L^{2}}^{2}\right\}+\mu \int_{0}^{t}\left\|D\left(v^{\varepsilon}\right)(\tau)\right\|_{L^{2}\left(\Omega_{\varepsilon}\right)}^{2} d \tau \\
\leq\left\{\frac{R^{4} \varepsilon^{4}}{16 \mu L} \int_{0}^{t}|A(\tau)|^{2} d \tau+\omega^{\varepsilon} \frac{\varepsilon^{3}}{h(\varepsilon) E(\varepsilon)} \frac{(1+\sigma) L R^{3}}{2}\|A(t)\|_{\mathcal{H}}^{2}\right\} e^{t}
\end{gathered}
$$

To capture the elastic response of the membrane to the oscillations in the pressure drop between the inlet and the outlet boundary, $\omega^{\varepsilon}$ is chosen so that both terms on the right-hand side are of the same order in $\epsilon$. Using assumption (2.4), we get

$$
\omega^{\varepsilon}=\frac{\varepsilon^{2}}{\mu}
$$

We are now ready to obtain the a priori solution estimates in terms of $\epsilon$. In the text that follows we denote all the constants independent of $\varepsilon$ by $C$. Define

$$
\|A\|_{\mathcal{V}}^{2}=e^{T}\left\{\|A\|_{L^{\infty}(0, T)}^{2}+\int_{0}^{T}\left(\left|\partial_{\tau} A(\tau)\right|^{2}+|A(\tau)|^{2}\right) d \tau\right\}
$$

Proposition 3.8. Solution $\left(v_{r}^{\varepsilon}, v_{z}^{\varepsilon}, \eta^{\varepsilon}, s^{\varepsilon}\right)$ of problem (3.1)-(3.2) satisfies the a priori estimates

$$
\int_{0}^{t}\left\{\left\|\frac{\partial v_{z}^{\varepsilon}}{\partial r}\right\|_{L^{2}\left(\Omega_{\varepsilon}\right)}^{2}+\left\|\frac{\partial v_{r}^{\varepsilon}}{\partial z}\right\|_{L^{2}\left(\Omega_{\varepsilon}\right)}^{2}\right\} d \tau \leq C\left\{\left(\frac{\varepsilon^{2}}{\mu}\right)^{2}\|A\|_{\mathcal{V}}^{2}+\varepsilon^{2}\left(\omega^{\varepsilon}\right)^{2} \int_{0}^{t}\left\|\partial_{\tau} s^{\varepsilon}(\tau)\right\|_{L^{2}(0, L)}^{2} d \tau\right\},
$$

$$
\int_{0}^{t}\left\{\left\|\frac{\partial v_{r}^{\varepsilon}}{\partial r}\right\|_{L^{2}\left(\Omega_{\varepsilon}\right)}^{2}+\left\|\frac{v_{r}^{\varepsilon}}{r}\right\|_{L^{2}\left(\Omega_{\varepsilon}\right)}^{2}+\left\|\frac{\partial v_{z}^{\varepsilon}}{\partial z}\right\|_{L^{2}\left(\Omega_{\varepsilon}\right)}^{2}\right\} d \tau \leq C\left(\frac{\varepsilon^{2}}{\mu}\right)^{2}\|A\|_{\mathcal{V}}^{2}
$$

Proof. First notice that (3.12) and (3.15) are obvious consequences of Proposition 3.7. Equation (3.14) is a variant of the Poincaré inequality, and so we need only to prove (3.13).

To prove (3.13) we start from estimate (3.10) for the shear stress term in $D\left(v^{\varepsilon}\right)$. It reads

$$
\int_{0}^{t} \int_{0}^{L} \int_{0}^{\varepsilon R}\left\{\left(\frac{\partial v_{r}^{\varepsilon}}{\partial z}\right)^{2}+2 \frac{\partial v_{r}^{\varepsilon}}{\partial z} \frac{\partial v_{z}^{\varepsilon}}{\partial r}+\left(\frac{\partial v_{z}^{\varepsilon}}{\partial r}\right)^{2}\right\} r d r d z d \tau \leq C\left(\frac{\varepsilon^{2}}{\mu}\right)^{2}\|A\|_{\mathcal{V}}^{2}
$$

The difficulty comes from the term which is the product of two off-diagonal gradient terms $\frac{\partial v_{r}^{\varepsilon}}{\partial z} \frac{\partial v_{z}^{\varepsilon}}{\partial r}$. Estimate this term by using the boundary behavior of $v^{\varepsilon}, \partial_{z} v_{z}^{\varepsilon}=0$ at $z=0, L$, and 
the incompressibility condition (2.9) to obtain

$$
\begin{aligned}
\int_{\Omega_{\varepsilon}} \frac{\partial v_{r}^{\varepsilon}}{\partial z} \frac{\partial v_{z}^{\varepsilon}}{\partial r} r d r d z & =-\int_{\Omega_{\varepsilon}}\left(v_{z}^{\varepsilon}-\omega^{\varepsilon} \partial_{t} s^{\varepsilon}\right) \frac{\partial}{\partial r}\left(r \frac{\partial v_{r}^{\varepsilon}}{\partial z}\right) d r d z \\
& =\int_{\Omega_{\varepsilon}}\left(v_{z}^{\varepsilon}-\omega^{\varepsilon} \partial_{t} s^{\varepsilon}\right) \frac{\partial^{2} v_{z}^{\varepsilon}}{\partial z^{2}} r d r d z \\
& =-\int_{\Omega_{\varepsilon}} \frac{\partial}{\partial z}\left(v_{z}^{\varepsilon}-\omega^{\varepsilon} \partial_{t} s^{\varepsilon}\right) \frac{\partial v_{z}^{\varepsilon}}{\partial z} r d r d z \\
& =-\int_{\Omega_{\varepsilon}}\left(\frac{\partial v_{z}^{\varepsilon}}{\partial z}\right)^{2} r d r d z+\omega^{\varepsilon} \int_{\Omega_{\varepsilon}} \frac{\partial}{\partial z}\left(\partial_{t} s^{\varepsilon}\right) \frac{\partial v_{z}^{\varepsilon}}{\partial z} r d r d z
\end{aligned}
$$

The rest of the proof is now immediate.

Corollary 3.9. The time derivatives $\partial_{t}\left(v_{r}^{\varepsilon}, v_{z}^{\varepsilon}, \eta^{\varepsilon}, s^{\varepsilon}\right)$ satisfy all the above estimates but with $A$ replaced by $\partial_{t} A$.

The important estimates are summarized in the following theorem. Here we recall Assumption 1 and use, with a slight abuse of notation, $E_{0}$ and $G_{0}$ to denote the expressions $E(\varepsilon) h(\varepsilon) / \varepsilon$ and $G(\varepsilon) k(\varepsilon) h(\varepsilon) \varepsilon$, respectively.

Theorem 3.10. Solution $\left(v_{r}^{\varepsilon}, v_{z}^{\varepsilon}, \eta^{\varepsilon}, s^{\varepsilon}\right)$ of problem (3.1)-(3.2) satisfies

$$
\begin{gathered}
\frac{G_{0}}{\varepsilon^{2}}\left\|\frac{\partial \eta^{\varepsilon}}{\partial z}(t)\right\|_{L^{2}(0, L)}^{2}+\frac{1}{\varepsilon^{2}}\left\|\eta^{\varepsilon}(t)\right\|_{L^{2}(0, L)}^{2}+\left\|\frac{\partial s^{\varepsilon}}{\partial z}(t)\right\|_{L^{2}(0, L)}^{2} \leq C \frac{1}{E_{0}}\|A\|_{\mathcal{V}}^{2}, \\
\left\|\frac{\partial v_{z}^{\varepsilon}}{\partial r}\right\|_{L^{2}\left(\Omega_{\varepsilon} \times(0, T)\right)}^{2}+\left\|\frac{\partial v_{r}^{\varepsilon}}{\partial z}\right\|_{L^{2}\left(\Omega_{\varepsilon} \times(0, T)\right)}^{2} \leq C\left(\frac{\varepsilon^{2}}{\mu}\right)^{2}\|A\|_{\mathcal{V}}^{2}, \\
\left\|v_{r}^{\varepsilon}\right\|_{L^{2}\left(\Omega_{\varepsilon} \times(0, T)\right)} \leq C \frac{\varepsilon^{3}}{\mu}\|A\|_{\mathcal{V}}, \\
\left\|v_{z}^{\varepsilon}\right\|_{L^{2}\left(\Omega_{\varepsilon} \times(0, T)\right)} \leq C \frac{\varepsilon^{3}}{\mu}\|A\|_{\mathcal{V}} .
\end{gathered}
$$

The same estimates hold for $\partial_{t}\left(v_{r}^{\varepsilon}, v_{z}^{\varepsilon}, \eta^{\varepsilon}, s^{\varepsilon}\right)$ but in terms of $\partial_{t} A$.

4. The rescaled problem and asymptotic expansions. In order to study problem $\mathrm{P}^{\varepsilon}$ in the limit as $\varepsilon \rightarrow 0$, it is convenient to use a rescaling which maps domain $\Omega_{\varepsilon}$ to a fixed domain $\Omega=\Omega_{1}$ corresponding to $\varepsilon=1$. This, in turn, rescales the variables and their derivatives in the following manner.

Let $u^{\varepsilon}$ be a sequence of axially symmetric functions defined on $\Omega_{\varepsilon}$ with values in $\mathbb{R}^{3}$, $u^{\varepsilon}=u_{r}^{\varepsilon} \vec{e}_{r}+u_{z}^{\varepsilon} \vec{e}_{z}$. Introduce the following scaling [14]:

$$
u(\varepsilon)(r, z)=u^{\varepsilon}(\varepsilon r, z) .
$$

The sequence $\{u(\varepsilon)\}$ is now a sequence of functions defined on a fixed domain $\Omega \equiv \Omega_{1}$. The 
rescaled differential operators $D_{\varepsilon}$ and $\operatorname{div}_{\varepsilon}$ applied on $u(\varepsilon)$ are given by

$$
\begin{gathered}
D_{\varepsilon}(u)_{r r}=\frac{1}{\varepsilon} \frac{\partial u_{r}}{\partial r}, \quad D_{\varepsilon}(u)_{\vartheta \vartheta}=\frac{u_{r}}{\varepsilon r}, \quad D_{\varepsilon}(u)_{r \vartheta}=0, \quad D_{\varepsilon}(u)_{z z}=\frac{\partial u_{z}}{\partial z}, \\
D_{\varepsilon}(u)_{r z}=\frac{1}{2}\left(\frac{\partial u_{r}}{\partial z}+\frac{1}{\varepsilon} \frac{\partial u_{z}}{\partial r}\right), \quad D_{\varepsilon}(u)_{z \vartheta}=0, \quad \text { and } \\
\operatorname{div}_{\varepsilon} u=\frac{1}{\varepsilon} \frac{\partial u_{r}}{\partial r}+\frac{\partial u_{z}}{\partial z}+\frac{1}{\varepsilon} \frac{u_{r}}{r} .
\end{gathered}
$$

The rescaled incompressible Stokes' equations (2.7), (2.8), and (2.9), defined on $\Omega \times \mathbb{R}_{+}$, read

$$
\begin{array}{r}
-\frac{\mu}{\varepsilon^{2}}\left(\frac{\partial^{2} v(\varepsilon)_{r}}{\partial r^{2}}+\varepsilon^{2} \frac{\partial^{2} v(\varepsilon)_{r}}{\partial z^{2}}+\frac{1}{r} \frac{\partial v(\varepsilon)_{r}}{\partial r}-\frac{v(\varepsilon)_{r}}{r^{2}}\right)+\frac{1}{\varepsilon} \frac{\partial p(\varepsilon)}{\partial r}=0 \\
-\frac{\mu}{\varepsilon^{2}}\left(\frac{\partial^{2} v(\varepsilon)_{z}}{\partial r^{2}}+\varepsilon^{2} \frac{\partial^{2} v(\varepsilon)_{z}}{\partial z^{2}}+\frac{1}{r} \frac{\partial v(\varepsilon)_{z}}{\partial r}\right)+\frac{\partial p(\varepsilon)}{\partial z}=0 \\
\varepsilon \operatorname{div}_{\varepsilon} v(\varepsilon)=\frac{\partial v(\varepsilon)_{r}}{\partial r}+\varepsilon \frac{\partial v(\varepsilon)_{z}}{\partial z}+\frac{v(\varepsilon)_{r}}{r}=0 .
\end{array}
$$

Since the quantities defined on the lateral boundary are invariant under this scaling, we use the same notation for the wall displacements of the rescaled problem as for the original problem, namely, $\eta^{\varepsilon}$ and $s^{\varepsilon}$. The lateral boundary conditions then read

$$
\begin{gathered}
\frac{\mu}{\varepsilon^{2}} v(\varepsilon)_{r}=\frac{\partial \eta^{\varepsilon}}{\partial t}, \quad \frac{\mu}{\varepsilon^{2}} v(\varepsilon)_{z}=\frac{\partial s^{\varepsilon}}{\partial t}, \\
\left(p(\varepsilon) I-2 \mu D_{\epsilon}(v(\varepsilon))\right) \vec{e}_{r} \cdot \vec{e}_{r}=-F_{r}, \\
\left(p(\varepsilon) I-2 \mu D_{\epsilon}(v(\varepsilon))\right) \vec{e}_{r} \cdot \vec{e}_{z}=-F_{z},
\end{gathered}
$$

where $F_{r}$ and $F_{z}$ are given by (2.2) and (2.3), respectively. The initial conditions and the inlet and outlet boundary data are $(2.14),(2.15)-(2.17)$ written in terms of the rescaled quantities.

To write this problem in variational form, introduce the space of all test functions $\varphi=$ $\varphi_{r} \vec{e}_{r}+\varphi_{z} \vec{e}_{z}$ to be the space $V$ given by Definition 1 using $\epsilon=1$. Furthermore, let $\mathcal{V}$ be the space of rescaled functions on a fixed domain $\Omega$ defined by Definition 2 with $\epsilon=1$, with the incompressibility condition for the velocity replaced by $\operatorname{div}_{\varepsilon} v(\varepsilon)=0$ and with $\frac{\partial}{\partial t}$ replaced by $\frac{\mu}{\varepsilon^{2}} \frac{\partial}{\partial t}$. Let $\psi \in C_{0}^{\infty}(0, T)$ be a temporal test function. Then, after rewriting variational equality (3.1) in rescaled variables, multiplying (3.1) by $\psi(t)$, integrating with respect to time, and dividing by $\varepsilon^{2}$, we obtain the variational formulation of the rescaled problem specified in (4.7) where

$$
\begin{aligned}
\mathcal{E}_{\mu}(v, \varphi, \psi ; \varepsilon) & \equiv \int_{0}^{T} \int_{\Omega} \frac{\mu}{\varepsilon^{2}} \frac{\partial v(\varepsilon)_{z}}{\partial r} \frac{\partial \varphi_{z}}{\partial r} \psi(t) r d r d z d t \\
& +2 \varepsilon \int_{0}^{T} \int_{\Omega} \frac{\mu}{\varepsilon^{2}}\left(\frac{1}{2} \frac{\partial v(\varepsilon)_{r}}{\partial z} \frac{\partial \varphi_{z}}{\partial r}+\frac{\partial v(\varepsilon)_{r}}{\partial r} \frac{\partial \varphi_{r}}{\partial r}+\frac{v(\varepsilon)_{r} \varphi_{r}}{r^{2}}\right) \psi(t) r d r d z d t \\
& +2 \varepsilon^{2} \int_{0}^{T} \int_{\Omega} \frac{\mu}{\varepsilon^{2}}\left(\frac{1}{2} \frac{\partial v(\varepsilon)_{z}}{\partial r} \frac{\partial \varphi_{r}}{\partial z}+\frac{\partial v(\varepsilon)_{z}}{\partial z} \frac{\partial \varphi_{z}}{\partial z}\right) \psi(t) r d r d z d t \\
& +\varepsilon^{3} \int_{0}^{T} \int_{\Omega} \frac{\mu}{\varepsilon^{2}} \frac{\partial v(\varepsilon)_{r}}{\partial z} \frac{\partial \varphi_{r}}{\partial z} \psi(t) r d r d z d t
\end{aligned}
$$




$$
\begin{aligned}
\mathcal{E}_{\mathrm{el}}\left(\eta^{\varepsilon}, s^{\varepsilon}, \varphi, \psi ; \varepsilon\right) & \equiv R \int_{0}^{T} \int_{0}^{L}\left\{h(\varepsilon) G(\varepsilon) k(\varepsilon) \frac{\partial \eta^{\varepsilon}}{\partial z} \frac{\partial \varphi_{r}}{\partial z}+\frac{h(\varepsilon) E(\varepsilon)}{1-\sigma^{2}}\left(\frac{\sigma}{\varepsilon R} \frac{\partial s^{\varepsilon}}{\partial z}\right.\right. \\
& \left.\left.+\frac{\eta^{\varepsilon}}{\varepsilon^{2} R^{2}}\right) \varphi_{r}+\frac{h(\varepsilon) E(\varepsilon)}{\varepsilon\left(1-\sigma^{2}\right)}\left(\frac{\partial s^{\varepsilon}}{\partial z} \frac{\partial \varphi_{z}}{\partial z}-\frac{\sigma}{\varepsilon R} \frac{\partial \eta^{\varepsilon}}{\partial z} \varphi_{z}\right)\right\} \psi(t) d z d t \\
& +\frac{R \rho_{w} h(\varepsilon)\left(\omega^{\varepsilon}\right)^{2}}{\varepsilon} \int_{0}^{T} \frac{d^{2} \psi(t)}{d t^{2}} \int_{0}^{L}\left(\varepsilon \eta^{\varepsilon} \varphi_{r}+s^{\varepsilon} \varphi_{z}\right) d z d t
\end{aligned}
$$

and

$$
\mathcal{E}_{\mathrm{src}}(A, \varphi, \psi) \equiv \int_{0}^{T} \int_{0}^{R} A(t) \varphi_{z} \psi(t) r d r d t .
$$

Definition 4 (weak formulation of the rescaled problem $\mathrm{P}(\varepsilon)$ ). Function $\left(v(\varepsilon)_{r}, v(\varepsilon)_{z}, \eta^{\varepsilon}, s^{\varepsilon}\right.$ ) $\in \mathcal{V}$ is a weak solution of problem $\mathrm{P}(\varepsilon)$ if the following variational formulation is satisfied:

$$
\mathcal{E}_{\mu}(v, \varphi, \psi ; \varepsilon)+\mathcal{E}_{\mathrm{el}}\left(\eta^{\varepsilon}, s^{\varepsilon}, \varphi, \psi ; \varepsilon\right)=-\mathcal{E}_{\mathrm{src}}(A, \varphi, \psi) \quad \forall \psi \in \mathcal{D}\left(\mathbb{R}_{+}\right) \text {and } \forall \varphi \in V .
$$

The initial conditions at the lateral boundary are

$$
\eta^{\varepsilon}=s^{\varepsilon}=\frac{\partial \eta^{\varepsilon}}{\partial t}=\frac{\partial s^{\varepsilon}}{\partial t}=0 \quad \text { on } \quad \Sigma \times\{0\} .
$$

Later in the text we will also need the weak formulation which includes the pressure. For this purpose we consider the test functions $\varphi$ which are not divergence-free. Namely, denote by

$$
\begin{gathered}
V_{\text {div } \neq 0}=\left\{\varphi \in H^{1}(\Omega)^{3} \mid \varphi \text { is axially symmetric, }\left.\varphi_{r}\right|_{\Sigma},\left.\varphi_{z}\right|_{\Sigma} \in H^{1}(0, L),\right. \\
\left.\varphi_{z}(L, R)=\varphi_{r}(L, r)=\varphi_{r}(0, r)=0\right\} .
\end{gathered}
$$

Then the weak formulation of the problem, cast in terms of the velocity and pressure, reads as follows.

Definition 5 (weak formulation of $\mathrm{P}(\varepsilon)$ in the pressure-velocity form). Vector function $\left(v(\varepsilon)_{r}\right.$, $\left.v(\varepsilon)_{z}, \eta^{\varepsilon}, s^{\varepsilon}\right) \in \mathcal{V}$ and $\left.p(\varepsilon) \in L^{2}((0, T) \times \Omega)\right)$ form a weak solution of problem $\mathrm{P}(\varepsilon)$ if

$$
\begin{aligned}
& \mathcal{E}_{\mu}(v(\varepsilon), \varphi, \psi ; \varepsilon)-\int_{0}^{T} \int_{\Omega} p(\varepsilon)\left(\frac{\partial \varphi_{z}}{\partial z}+\frac{\partial \varphi_{r}}{\partial r}+\frac{\varphi_{r}}{r}\right) \psi(t) r d r d z d t \\
& +\mathcal{E}_{\mathrm{el}}\left(\eta^{\varepsilon}, s^{\varepsilon}, \varphi, \psi ; \varepsilon\right)=-\mathcal{E}_{\mathrm{src}}(A, \varphi, \psi) \quad \text { in } \mathcal{D}^{\prime}\left(\mathbb{R}_{+}\right) \forall \varphi \in V_{\text {div } \neq 0} .
\end{aligned}
$$

Proposition 4.1. Variational problem (3.1), (3.2) is equivalent to the rescaled problem (4.3), (4.7), and (4.8).

The a priori estimates for the rescaled functions follow from Proposition 3.8, Theorem 3.10, and the following result.

Lemma 4.2. For $v \in L^{2}\left(\Omega_{\varepsilon}\right)$ the rescaled function $v(\varepsilon)$ satisfies

$$
\begin{aligned}
\|v(\varepsilon)\|_{L^{2}(\Omega)} & =\varepsilon^{-1}\|v\|_{L^{2}\left(\Omega_{\varepsilon}\right)}, \\
\left\|\frac{\partial v(\varepsilon)}{\partial r}\right\|_{L^{2}(\Omega)} & =\left\|\frac{\partial v}{\partial r}\right\|_{L^{2}\left(\Omega_{\varepsilon}\right)}, \\
\left\|\frac{\partial v(\varepsilon)}{\partial z}\right\|_{L^{2}(\Omega)} & =\varepsilon^{-1}\left\|\frac{\partial v}{\partial z}\right\|_{L^{2}\left(\Omega_{\varepsilon}\right)} .
\end{aligned}
$$


Corollary 4.3. Solution $\left(v(\varepsilon)_{r}, v(\varepsilon)_{z}, \eta^{\varepsilon}, s^{\varepsilon}\right)$ of the rescaled problem satisfies the a priori estimates

$$
\begin{gathered}
\left\|\frac{v(\varepsilon)_{r}}{r}\right\|_{L^{2}}+\left\|v(\varepsilon)_{z}\right\|_{L^{2}}+\left\|\frac{\partial v(\varepsilon)_{r}}{\partial r}\right\|_{L^{2}}+\left\|\frac{\partial v(\varepsilon)_{z}}{\partial r}\right\|_{L^{2}} \leq C \frac{\varepsilon^{2}}{\mu}\|A\|_{\mathcal{V}} \\
\left\|\frac{\partial v(\varepsilon)_{r}}{\partial z}\right\|_{L^{2}}+\left\|\frac{\partial v(\varepsilon)_{z}}{\partial z}\right\|_{L^{2}} \leq C \frac{\varepsilon}{\mu}\|A\|_{\mathcal{V}} \\
\left\|s^{\varepsilon}\right\|_{H^{1}} \leq C\|A\|_{\mathcal{V}}
\end{gathered}
$$

where all the norms are defined on $\Omega \times(0, T)$.

From the rescaled momentum equation relating the gradient of the pressure with the viscous effects of the fluid, we get an a priori estimate for the pressure $p(\varepsilon)$ corresponding to $v(\varepsilon)$. More precisely, we have the following proposition.

Proposition 4.4. The scaled pressure $p(\varepsilon)$ satisfies the estimates

$$
\begin{gathered}
\|p(\varepsilon)\|_{L^{2}(\Omega \times(0, T))} \leq C\|A\|_{\mathcal{V}} \\
\int_{0}^{T}\left\|\frac{\partial}{\partial z} p(\varepsilon)\right\|_{H^{-1}(\Omega)}^{2} d t+\frac{1}{\varepsilon^{2}} \int_{0}^{T}\left\|\frac{\partial}{\partial r} p(\varepsilon)\right\|_{H^{-1}(\Omega)}^{2} d t \leq C\|A\|_{\mathcal{V}}^{2} .
\end{gathered}
$$

Proof. Let $\varphi$ be an axially symmetric function in $H^{1}(\Omega)^{3}$ such that $\varphi=0$ on $\partial \Omega \backslash\{z=0\}$. Then, for all such $\varphi$ the scaled momentum equation gives

$$
\begin{aligned}
\left\langle\nabla_{\epsilon} p(\varepsilon), \varphi\right\rangle_{\Omega} & =\left\langle\frac{1}{\varepsilon} \frac{\partial}{\partial r} p(\varepsilon), \varphi_{r}\right\rangle_{\Omega}+\left\langle\frac{\partial}{\partial z} p(\varepsilon), \varphi_{z}\right\rangle_{\Omega} \\
& =-\mu \int_{\Omega} D_{\varepsilon}(v(\varepsilon)): D_{\varepsilon}(\varphi) .
\end{aligned}
$$

Now let $g \in L^{2}(\Omega)$. Due to the surjectivity of the divergence operator between any subspace of $H^{1}(\Omega)^{3}$ bigger than $H_{0}^{1}(\Omega)^{3}$ and $L^{2}(\Omega)$, there exists a $\tilde{\varphi} \in H^{1}(\Omega)^{3}$, axially symmetric with $\tilde{\varphi}=0$ on $\partial \Omega \backslash\{z=0\}$, such that $\operatorname{div} \tilde{\varphi}=g$. Let $\varphi_{r}=\varepsilon \tilde{\varphi}_{r}$ and $\varphi_{z}=\tilde{\varphi}_{z}$. Then $g=\operatorname{div}_{\varepsilon} \varphi$ and $\left|\int_{\Omega} p(\varepsilon) g\right|=\left|\int_{\Omega} p(\varepsilon) \operatorname{div}_{\varepsilon} \phi\right|=\left|\left\langle\nabla_{\epsilon} p(\varepsilon), \varphi\right\rangle_{\Omega}\right| \leq C\|g\|_{L^{2}(\Omega)} \forall g \in L^{2}(\Omega)$, implying (4.18).

After obtaining the uniform estimates for $\{v(\varepsilon), p(\varepsilon)\}$, which are valid for their time derivatives as well, we are in a situation where we can define the "correct" asymptotic expansions for $v(\varepsilon), p(\varepsilon), \eta^{\varepsilon}$, and $s^{\varepsilon}$. The usual difficulty with asymptotic expansions is to determine the "optimal" choice of the leading order powers of $\varepsilon$. In general, they follow from the a priori estimates. More precisely, for problem (3.1)-(3.2), results from Corollary 4.3 and Proposition 4.4 imply that the $L^{2}$-norms of $\frac{\mu}{\varepsilon^{2}} v(\varepsilon)$ and $p(\varepsilon)$ are bounded independently of $\varepsilon$ and $\mu$. Thus it is natural to use the following asymptotic expansions:

$$
\begin{gathered}
v(\varepsilon)(z, r, t)=\frac{\varepsilon^{2}}{\mu} \sum_{i \geq 0} \varepsilon^{i} v^{i}(z, r, t), \\
p(\varepsilon)(z, r, t)=\sum_{i \geq 0} \varepsilon^{i} p^{i}(z, r, t),
\end{gathered}
$$




$$
\begin{aligned}
\eta^{\varepsilon}(z, t) & =\varepsilon \sum_{i \geq 0} \varepsilon^{i} \eta^{i}(z, t), \\
s^{\varepsilon}(z, t) & =\sum_{i \geq 0} \varepsilon^{i} s^{i}(z, t) .
\end{aligned}
$$

In the next section we will use these expansions to derive the reduced problem, which, as we shall see, will be second-order accurate in $\epsilon$. In section 6.1 we prove the existence of a unique solution of the limiting problem, as $\epsilon \rightarrow 0$. This shows, in particular, that the a priori estimates and the asymptotic expansions given above are optimal.

5. The reduced problem. We derive the effective (reduced) equations, second-order accurate in $\varepsilon$, that hold for small $\varepsilon$. The reduced equations are obtained by inserting asymptotic expansions above into (4.1), (4.2), and (4.3) and by collecting the powers of $\varepsilon$. The incompressibility condition (4.3) implies

$$
\varepsilon^{-1} \frac{\partial}{r \partial r}\left(r v_{r}^{0}\right)+\frac{\partial v_{z}^{0}}{\partial z}+\frac{\partial}{r \partial r}\left(r v_{r}^{1}\right)+\varepsilon \sum_{i \geq 0} \varepsilon^{i}\left\{\frac{\partial v_{z}^{i+1}}{\partial z}+\frac{\partial}{r \partial r}\left(r v_{r}^{i+2}\right)\right\}=0
$$

Relation (5.1) gives

$$
\begin{aligned}
& v_{r}^{0}=0 \text { and } \\
& \frac{\partial\left(v_{z}^{0}+\varepsilon v_{z}^{1}\right)}{\partial z}+\frac{\partial}{r \partial r}\left(r\left(v_{r}^{1}+\varepsilon v_{r}^{2}\right)\right)=0 \quad \text { in } \Omega \times(0, T) .
\end{aligned}
$$

Notice that (5.2) indicates that in this coupled fluid-structure problem for creeping flow, the radial component of the velocity is by one order of magnitude smaller than the axial component.

Insert expansions (4.19)-(4.22) into the momentum equations (4.1) and (4.2), and collect the powers of $\varepsilon$ to obtain

$$
\begin{gathered}
-\frac{1}{\varepsilon} \frac{\partial\left(p^{0}+\varepsilon p^{1}\right)}{\partial r}+\varepsilon\left\{\frac{\partial}{r \partial r}\left(r \frac{\partial v_{r}^{1}}{\partial r}\right)-\frac{v_{r}^{1}}{r^{2}}-\frac{\partial p^{2}}{\partial r}\right\}=\mathcal{O}\left(\varepsilon^{2}\right), \\
\frac{\partial}{r \partial r}\left(r \frac{\partial\left(v_{z}^{0}+\varepsilon v_{z}^{1}\right)}{\partial r}\right)-\frac{\partial\left(p^{0}+\varepsilon p^{1}\right)}{\partial z}=\mathcal{O}\left(\varepsilon^{2}\right) .
\end{gathered}
$$

To simplify notation, define

$$
p=p^{0}+\varepsilon p^{1}, s=s^{0}+\varepsilon s^{1}, \eta=\eta^{0}+\varepsilon \eta^{1}, v_{r}=v_{r}^{1}+\varepsilon v_{r}^{2}, \quad \text { and } \quad v_{z}=v_{z}^{0}+\varepsilon v_{z}^{1} .
$$

The incompressibility condition (5.3) then reads

$$
\frac{\partial}{\partial r}\left(r v_{r}\right)+\frac{\partial}{\partial z}\left(r v_{z}\right)=0
$$

and the coefficient in front of $\varepsilon^{-1}$ in (5.4) implies

$$
p=p(z, t),
$$


which says that pressure is constant along each fixed cross-section of a vessel. The second momentum equation (5.5) implies

$$
r \frac{\partial p}{\partial z}=\frac{\partial}{\partial r}\left(r \frac{\partial v_{z}}{\partial r}\right)
$$

Equations (5.6), (5.7), and (5.8) are the standard asymptotic equations obtained from the flow equations before any boundary conditions are taken into account. These are obtained, for example, in [4, 20], using direct methods, in the case when the acceleration terms are not negligible. In that case, additional acceleration terms appear in (5.8). We mention that, typically, the viscous term on the right-hand side of (5.8) contains a factor with viscosity $\mu$. In our reduction, this term is hidden by its inclusion in the asymptotic expansion of the velocity (4.19). What is typically done next in the reduction to a one-dimensional model is the averaging of the flow equations over a fixed cross-sectional area. This is where boundary conditions and modeling of vessel walls are included. In the case frequently studied in literature, the wall (structure) is modeled using the "independent ring model" [3, 4, 20, 10, 11, 22]. In that case the longitudinal displacement $s$ is assumed to be zero, and the shear term $\partial \eta / \partial z$ is assumed negligible. The no-slip boundary condition is used at the lateral boundary.

In this paper, we do not use the no-slip condition, but we use the continuity of forces and velocity at the lateral boundary, which is more realistic when compliant vessel walls are considered. The structure (wall) is modeled by (2.2) and (2.3), whose asymptotic form is given by

$$
\begin{gathered}
p(z, t)=\frac{E_{0}}{R\left(1-\sigma^{2}\right)}\left(\sigma \frac{\partial s}{\partial z}+\frac{\eta}{R}\right)-G_{0} \frac{\partial^{2} \eta}{\partial z^{2}}+\mathcal{O}\left(\varepsilon^{2}\right), \\
\left.\frac{\partial v_{z}}{\partial r}\right|_{r=R}=\frac{E_{0}}{1-\sigma^{2}} \frac{\partial}{\partial z}\left(\frac{\partial s}{\partial z}+\frac{\sigma \eta}{R}\right)+\mathcal{O}\left(\varepsilon^{2}\right) .
\end{gathered}
$$

Here again, with a slight abuse of notation, we used $E_{0}$ and $G_{0}$ to denote the expressions $E(\varepsilon) h(\varepsilon) / \varepsilon$ and $G(\varepsilon) k(\varepsilon) h(\varepsilon) \varepsilon$, respectively. It turns out that the reduced equations in this case are much easier to write in terms of the effective pressure, $p$, rather than in terms of the cross-sectional area and the volumetric flow rate which are typically used when the independent ring model is employed. With this in mind, we proceed as follows. We focus on (5.9) and (5.10) to obtain the PDE for the pressure. We eliminate $\eta, v_{z}$, and $\frac{\partial s}{\partial z}$ from (5.9) and (5.10) by using the incompressibility condition (5.6) and the momentum equations (5.7) and (5.8) in the following way. Integrate the leading order term in the second momentum equation (5.8) twice, from $r$ to $R$, and use

$$
v_{z}(z, R, t)=\frac{\partial s}{\partial t}(z, t)
$$

to obtain the axial velocity

$$
v_{z}(z, r, t)=\frac{r^{2}-R^{2}}{4} \frac{\partial p}{\partial z}(z, t)+\frac{\partial s}{\partial t}(z, t) .
$$


This is a variant of the Biot law for velocity; see [1, 24]. Combined with the incompressibility condition it provides the following relation used in the proof of the convergence theorem (see section 6$)$ :

$$
v_{r}=\frac{R}{r} \frac{\partial \eta}{\partial t}-\frac{\left(R^{2}-r^{2}\right)^{2}}{16 r} \frac{\partial^{2} p}{\partial z^{2}}+\frac{R^{2}-r^{2}}{2 r} \frac{\partial^{2} s}{\partial z \partial t}=\frac{r}{2} \frac{\partial}{\partial z}\left\{\frac{2 R^{2}-r^{2}}{8} \frac{\partial p}{\partial z}-\frac{\partial s}{\partial t}\right\} .
$$

Next use (5.12) in (5.10) to eliminate $v_{z}$ by observing that

$$
\left.\frac{\partial v_{z}}{\partial r}\right|_{r=R}=\frac{R}{2} \frac{\partial p}{\partial z}(z, t)
$$

To eliminate $\frac{\partial s}{\partial z}$, plug expression (5.12) for $v_{z}$ into the reduced incompressibility condition (5.6) integrated from $r=0$ to $r=R$,

$$
\frac{\partial \eta}{\partial t}+\frac{\partial}{\partial z}\left(\frac{1}{R} \int^{R} v_{z}(r, z, t) r d r\right)=0
$$

to obtain the reduced, averaged, incompressibility condition

$$
\frac{\partial \eta}{\partial t}-\frac{R^{3}}{16} \frac{\partial^{2} p}{\partial z^{2}}+\frac{R}{2} \frac{\partial^{2} s}{\partial z \partial t}=0
$$

Integrate with respect to $t$, and obtain

$$
\frac{\partial s}{\partial z}=\frac{R^{2}}{8} \frac{\partial^{2}}{\partial z^{2}} \int_{0}^{t} p-\frac{2}{R} \eta
$$

Insert (5.13) into (5.10) to obtain

$$
\frac{R}{2} p(z, t)-\frac{E_{0}}{1-\sigma^{2}}\left(\frac{\partial s}{\partial z}+\frac{\sigma \eta}{R}\right)=C_{0}(t) .
$$

We claim that $C_{0}(t)=C_{0}=0$. To see this, consider even extensions of the axial velocity $v_{z}^{\varepsilon}$ and the axial displacement $s^{\varepsilon}$ for negative values of $z$. Analogously, make odd extensions of the radial velocity $v_{r}^{\varepsilon}$, radial displacement $\eta^{\varepsilon}$, and the pressure $p^{\varepsilon}$. It is easy to check that all the equations are valid for $z \in(-L, L)$. Consequently, the partial derivatives with respect to $z$ of all the quantities have the same regularity as the unknown functions. Hence

$$
\eta(0, t)=0=p(0, t)=\frac{\partial s}{\partial z}(0, t) .
$$

Therefore, $C_{0}(t)=0$.

From this calculation we obtain the following relation between $\eta$ and $p$ :

$$
\frac{E_{0}}{R\left(1-\sigma^{2}\right)} \eta=\frac{R}{2-\sigma}\left(-\frac{1}{2} p+\frac{E_{0} R}{8\left(1-\sigma^{2}\right)} \frac{\partial^{2}}{\partial z^{2}} \int_{0}^{t} p\right)
$$

We use this to eliminate $\eta$ from (5.9) and (5.10). 
Note that at this point we can write (5.9) and (5.10) entirely in terms of the pressure: (5.18) relates $\eta$ with $p,(5.16)$ relates $\frac{\partial s}{\partial z}$ with $\eta$, and therefore with $p$, and (5.12) implies that the second equation (5.10) reads

$$
\frac{\partial p}{\partial z}(z, t)=\frac{2}{R}\left(\frac{E_{0}}{1-\sigma^{2}} \frac{\partial}{\partial z}\left(\frac{\partial s}{\partial z}+\frac{\sigma \eta}{R}\right)\right)+\mathcal{O}\left(\varepsilon^{2}\right) .
$$

By differentiating the pressure equation (5.9) with respect to $z$ and by setting it equal to (5.19), we obtain the reduced equation for the pressure

$$
\frac{\partial}{\partial t}\left\{\left(\frac{5}{2}-2 \sigma\right) p-\left(1-\sigma^{2}\right) \frac{G_{0} R^{2}}{2 E_{0}} \frac{\partial^{2} p}{\partial z^{2}}\right\}=\frac{\partial^{2}}{\partial z^{2}}\left\{\frac{E_{0} R}{8} p-\frac{G_{0} R^{3}}{8} \frac{\partial^{2} p}{\partial z^{2}}\right\} .
$$

Remark 1. Note that the "correct" time-scale for the pressure is

$$
t_{p}=\frac{E_{0} R_{\tilde{t}}}{8}=\left(\lim _{\varepsilon \rightarrow 0} \frac{h(\varepsilon) E(\varepsilon) \varepsilon R}{8 \mu}\right) t
$$

It is interesting to notice that for the values given in Table 2.1 this is 0.75 of the time-scale $\tilde{t}$ and 0.35 of the original physical time-scale $t$. The physical time-scale $t$ and the time-scale $\tilde{t}$ are related via $\tilde{t}=\left(\varepsilon^{2} / \mu\right) t=0.47 t$.

Depending on the problem, the coefficients containing shear modulus $G_{0}$ may or may not be negligible. In the two subsections that follow, we summarize the initial-boundary-value problems corresponding to the two cases.

5.1. The reduced problem with small shear modulus. In this subsection we study the case when the coefficients containing shear modulus $G_{0}$ are zero or negligible. After taking into account the obvious regularity of $p^{\varepsilon}$ with respect to $z$ (but not necessarily the regularity of $s^{\varepsilon}$ ), we see that $p=A(t)$ for $z=L$. The reduced initial-boundary-value problem for the effective pressure reads

$$
\left\{\begin{array}{l}
\left(\frac{5}{2}-2 \sigma\right) \frac{\partial p}{\partial t}=\frac{E_{0} R}{8} \frac{\partial^{2} p}{\partial z^{2}} \quad \text { in } \quad(0, L) \times(0, T), \\
p(0, t)=0, \quad p(L, t)=A(t) \quad \text { in } \quad(0, T), \\
p(z, 0)=0 \quad \text { in } \quad(0, L),
\end{array}\right.
$$

and the relationship between $\frac{\partial s}{\partial z}$ and $\eta$ becomes

$$
\frac{\partial s}{\partial z}=\frac{1-2 \sigma}{2-\sigma} \frac{\eta}{R}
$$

There is a unique smooth solution of problem (5.21). For incompressible materials, $\sigma=1 / 2$ [23], in which case (5.22) implies $s=0$ and

$$
p=\frac{4 E_{0}}{3 R^{2}} \eta=\frac{4 E_{0}}{3 R}\left(1-\sqrt{\frac{\mathcal{A}(0)}{\mathcal{A}}}+\mathcal{O}\left(\frac{\eta}{R}\right)\right),
$$


where $\mathcal{A}=\mathcal{A}(x, t)$ denotes the cross-sectional area at $(t, x)$, and $\mathcal{A}(0)$ is the unstressed crosssectional area at $x$ (corresponding to the zero pressure). This is the law of Laplace, or the independent ring model, found in [11, 20] using different approaches. In general, for negligible shear modulus, we find that pressure is directly related to the radial displacement via

$$
p=\frac{E_{0} \eta}{(1-\sigma / 2) R^{2}}=\frac{E_{0}}{(1-\sigma / 2) R}\left(1-\sqrt{\frac{\mathcal{A}(0)}{\mathcal{A}}}+\mathcal{O}\left(\frac{\eta}{R}\right)\right),
$$

where (5.23) is the first term in the expansion for the pressure in terms of the radial displacement. We see that, for general $\sigma$, the diffusion equation for the effective pressure can be easily written in terms of the radial displacement. The resulting equation is again parabolic, reflecting the fact that acceleration terms in the fluid equation have been ignored. More precisely, if the acceleration terms were present (Navier-Stokes equations), the resulting equation would include the second derivative of $\eta$ with respect to time and give a hyperbolic problem. Hyperbolic problems are typically obtained when the reduced Navier-Stokes equations are coupled with the independent ring model $[12,20,4]$.

Remark 2. If $A \in C_{0}^{\infty}(0,+\infty)$, then $p \in C^{\infty}([0, L] \times[0, T])$.

5.2. The reduced problem for nonnegligible shear modulus. In the case when the shear modulus coefficients are not small, we need more boundary conditions for (5.20). Furthermore, for $G_{0}>0$ the boundary conditions for the radial displacement are preserved in the limit. By using (5.18) we get the boundary conditions for $\partial_{z z} p$ at $z=0, L$. The reduced initialboundary-value problem reads

$$
\left\{\begin{array}{l}
\frac{\partial}{\partial t}\left\{\left(\frac{5}{2}-2 \sigma\right) p-\left(1-\sigma^{2}\right) \frac{G_{0} R^{2}}{2 E_{0}} \frac{\partial^{2} p}{\partial z^{2}}\right\}=\frac{\partial^{2}}{\partial z^{2}}\left\{\frac{E_{0} R}{8} p-\frac{G_{0} R^{3}}{8} \frac{\partial^{2} p}{\partial z^{2}}\right\} \\
p(0, t)=0, \quad p(L, t)=A(t) \quad \text { in } \quad(0, T), \\
\frac{\partial^{2} p}{\partial z^{2}}(0, t)=0, \quad \frac{E_{0} R}{8\left(1-\sigma^{2}\right)} \frac{\partial^{2} p}{\partial z^{2}}(L, t)=\frac{1}{2} \frac{d A}{d t} \quad \text { in } \quad(0, T), \\
p(z, 0)=0 \quad \text { in } \quad(0, L) .
\end{array}\right.
$$

Remark 3. If $A \in C_{0}^{\infty}(0,+\infty)$, then $p \in C^{\infty}([0, L] \times[0, T])$.

5.3. The reduced problem in the pressure-velocity form. It is useful to cast the above reduced problem in terms of the leading order velocity and pressure. In fact, in section 6 we will show that the solution of the original problem converges to the solution of the reduced problem written in terms of $\left(v_{z}, p, \eta, s\right)$. For this purpose we define the reduced problem for $\left(v_{z}, p, \eta, s\right)$ by using (5.14) and (5.8) to describe conservation of mass and momentum, (5.11), (5.9), and (5.10) to describe the lateral boundary conditions, and conditions corresponding to (2.15), (2.16), and (2.17) as the inlet and outlet boundary data. Summarized, the problem can be written as follows. Find $\left(v_{z}, p, \eta, s\right)$ such that the following equations describing conservation of mass and momentum hold:

$$
\begin{gathered}
\frac{\partial \eta}{\partial t}+\frac{\partial}{\partial z}\left(\frac{1}{R} \int^{R} v_{z}(r, z, t) r d r\right)=0, \\
r \frac{\partial p}{\partial z}=\frac{\partial}{\partial r}\left(r \frac{\partial v_{z}}{\partial r}\right)
\end{gathered}
$$


the lateral boundary conditions are

$$
\begin{gathered}
v_{z}(z, R, t)=\frac{\partial s}{\partial t}(z, t) \\
p(z, t)=\frac{E_{0}}{R\left(1-\sigma^{2}\right)}\left(\sigma \frac{\partial s}{\partial z}+\frac{\eta}{R}\right)-G_{0} \frac{\partial^{2} \eta}{\partial z^{2}} \\
\left.\frac{\partial v_{z}}{\partial r}\right|_{r=R}=\frac{E_{0}}{1-\sigma^{2}} \frac{\partial}{\partial z}\left(\frac{\partial s}{\partial z}+\frac{\sigma \eta}{R}\right)
\end{gathered}
$$

and the inlet and outlet boundary data are given by

$$
\begin{gathered}
\eta(0, t)=0=p(0, t)=\frac{\partial s}{\partial z}(0, t) \\
\eta(L, t)=0=s(L, t)=0, \quad p(L, t)=A(t), \\
\eta(z, 0)=0=s(z, 0) .
\end{gathered}
$$

In the next section we show that the solution of the original problem converges to the solution of the above reduced problem. Furthermore, in section 7 we show that the error between the solution of this reduced problem and the solution of the full Stokes problem coupled with the Navier equations for the membrane is of order $\epsilon^{2}$ except at the outlet boundary, where, due to the existence of a boundary layer, the error increases to $\mathcal{O}\left(\epsilon^{3 / 2}\right)$.

6. Convergence theorem. In this section we study the rescaled problem $\mathrm{P}(\varepsilon)$ in the limit as $\varepsilon \rightarrow 0$. We show that there exists a subsequence of a sequence of solutions of the rescaled problem which converges weakly and that the weak limit (each cluster point) satisfies the weak form of the reduced problem presented in section 5.3. Due to the uniqueness of the solution of the reduced problem, we conclude that every convergent subsequence of the sequence of rescaled solutions converges weakly to the unique solution of the reduced problem.

We start by proving the following weak compactness result for the sequence of solutions of problem $\mathrm{P}(\varepsilon)$.

Let

$$
\begin{aligned}
& W=\left\{\varphi \in L^{2}(\Omega) \mid \frac{\partial \varphi}{\partial r} \in L^{2}(\Omega)\right\} \text { and } \\
& W_{r}=\left\{r^{-1} \varphi \in L^{2}(\Omega) \mid \frac{\partial \varphi}{\partial r} \in L^{2}(\Omega)\right\} .
\end{aligned}
$$

Theorem 6.1. There exists a subsequence $\left(v(\varepsilon), p(\varepsilon), \eta^{\varepsilon}, s^{\varepsilon}\right)$ chosen from a sequence of solutions of the rescaled problem $\mathrm{P}(\varepsilon)$ such that $\left(\mu / \varepsilon^{2} v(\varepsilon), p(\varepsilon), \eta^{\varepsilon}, s^{\varepsilon}\right)$ converges weakly as $\epsilon \rightarrow 0$. Denote its limit (cluster point) by $(u, p, \eta, s)$, where $u=u_{r} \vec{e}_{r}+u_{z} \vec{e}_{z}$. Then

$$
\begin{array}{rlrl}
u(\varepsilon)_{z} & =\frac{\mu}{\varepsilon^{2}} v(\varepsilon)_{z} \rightarrow u_{z} \quad & \text { weakly in } & L^{2}(0, T ; W), \\
u(\varepsilon)_{r} & =\frac{\mu}{\varepsilon^{2}} v(\varepsilon)_{r} \rightarrow u_{r} \quad \text { weakly in } \quad L^{2}\left(0, T ; W_{r}\right), \\
s^{\varepsilon} & \rightarrow s \quad \text { weakly in } & H^{1}((0, T) \times(0, L)),
\end{array}
$$




$$
\begin{aligned}
\sqrt{G_{0}} \varepsilon^{-1} \partial_{z} \eta^{\varepsilon} & \rightarrow \sqrt{G_{0}} \partial_{z} \eta, \\
\varepsilon^{-1} \eta^{\varepsilon} & \rightarrow \eta \\
p(\varepsilon) & \rightarrow p \quad \text { weakly in } \quad L^{2}((0, T) \times \Omega) .
\end{aligned} \text { weak }{ }^{*} \text { in } L^{\infty}\left(0, T ; L^{2}(0, L)\right),
$$

Moreover, $u_{r}=0, \frac{\partial p}{\partial r}=0$, and

$$
\frac{\partial \eta}{\partial t}+\frac{\partial}{\partial z}\left(\frac{1}{R} \int_{0}^{R} u_{z}(r, z, t) r d r\right)=0 \quad \text { in the sense of distributions. }
$$

Finally,

$$
\varepsilon^{-1} \partial_{t} \eta^{\varepsilon} \rightarrow \partial_{t} \eta \quad \text { weakly in } \quad L^{2}\left(0, T ; H^{-1}(0, L)\right)
$$

Proof. We need only to prove $u_{r}=0$ and $\frac{\partial p}{\partial r}=0$ and the conservation of mass equation (6.8).

To show $u_{r}=0$, multiply the incompressibility condition (4.3) by a test function $\varphi \in$ $C_{0}^{\infty}(\Omega)$ to obtain

$$
\int_{\Omega} \varphi \frac{\partial}{\partial r}\left(r u(\varepsilon)_{r}\right) d r d z=-\varepsilon \int_{\Omega} \frac{\partial \varphi}{\partial z} u(\varepsilon)_{z} r d r d z \rightarrow 0 \quad \text { as } \quad \varepsilon \rightarrow 0 .
$$

Therefore, $\frac{\partial}{\partial r}\left(r u_{r}\right)=0$, and, since $u_{r} \in W_{r}$, it follows that $u_{r}=0$.

To show that $\frac{\partial p}{\partial r}=0$, observe that (4.18) implies that for every $\xi \in L^{2}\left(0, T ; H_{0}^{1}(\Omega)\right)$ we have

$$
\begin{gathered}
\left|\int_{0}^{T}\left\langle\frac{\partial}{\partial r} p, \xi\right\rangle_{\Omega} d t\right|=\left|-\int_{0}^{T} \int_{\Omega} p \frac{\partial \xi}{\partial r} r d r d z d t\right| \\
=\left|-\lim _{\varepsilon \rightarrow 0} \int_{0}^{T} \int_{\Omega} p(\varepsilon) \frac{\partial \xi}{\partial r} r d r d z d t\right|=\left|\lim _{\varepsilon \rightarrow 0} \int_{0}^{T}\left\langle\frac{\partial}{\partial r} p(\varepsilon), \xi\right\rangle_{\Omega} d t\right| \\
\leq\left\{\lim _{\varepsilon \rightarrow 0}\left\|\frac{\partial}{\partial r} p(\varepsilon)\right\|_{L^{2}\left(0, T ; H^{-1}(\Omega)\right)}\right\}\|\xi\|_{L^{2}\left(0, T: H_{0}^{1}(\Omega)\right)}=0,
\end{gathered}
$$

and we conclude that $p$ is independent of $r$, namely $p=p(z, t)$.

To show that conservation of mass (6.8) holds, start with the incompressibility condition (4.3) and multiply (4.3) by the test functions $\varphi \in C_{0}^{\infty}(0, L)$ and $\psi \in C_{0}^{\infty}(0, T)$. Integrating over $\Omega$ implies

$$
-R \int_{0}^{T} \int_{0}^{L} \frac{\eta^{\varepsilon}}{\varepsilon} \varphi \frac{d \psi}{d t} d z d t-\int_{0}^{T} \int_{\Omega} u(\varepsilon)_{z} \psi(t) \frac{d \varphi}{d z} r d r d z d t=0 .
$$

After passing to the limit as $\varepsilon \rightarrow 0$, we obtain (6.8) and (6.9).

Corollary 6.2. Let $G_{0}>0$. Then for any cluster point $\left\{u_{z}, p, \eta, s\right\} \in L^{2}(0, T ; W) \times$ $L^{2}(\Omega \times(0, T)) \times\left(L^{\infty}\left(0, T ; H^{1}(0, L)\right)^{2} \cap C\left([0, T] ; L^{2}(0, L)\right)^{2}\right)$ we have

$$
\begin{gathered}
u_{z}(R, z, t)=\frac{\partial s}{\partial t}(z, t) \text { for }(z, t) \in(0, L) \times(0, T), \\
\eta(0, t)=0=p(0, t)=\frac{\partial s}{\partial z}(0, t) \text { for } t \in(0, T), \\
\eta(L, t)=0=s(L, t)=0, p(L, t)=A(t) \text { for } t \in(0, T), \\
s(z, 0)=\eta(z, 0)=0 \text { for } z \in(0, L) .
\end{gathered}
$$


For $G_{0}=0, \eta \in L^{\infty}\left(0, T ; L^{2}(0, L)\right)$, and we drop the boundary condition for $\eta$ at $z=L$.

Proof. Relation (6.10) is a consequence of the preceding theorem. To show the rest, make an even extension of the axial velocity $v_{z}^{\varepsilon}$ and the axial displacement $s^{\varepsilon}$ for negative values of $z$ and odd extension of the radial velocity $v_{r}^{\varepsilon}$, radial displacement $\eta^{\varepsilon}$, and pressure $p^{\varepsilon}$. It is easy to check that all the equations are valid for $z \in(-L, L)$. Consequently, the partial derivatives with respect to $z$ of all the quantities have the same regularity as the unknown functions, and we conclude that (6.11) holds. The boundary condition for the limit pressure in (6.12) is obtained in the same way. The boundary conditions for the displacements follow from the corresponding convergence results proved in Theorem 6.1. The initial conditions (6.13) for $s$ and $\eta$ follow from (6.5), (6.6), and (6.9).

Motivated by the weak formulation of the rescaled problem $\mathrm{P}(\varepsilon)$, assumptions $(2.5)$ and (2.6), and the weak compactness result above, we define the following weak formulation of the limiting problem; call it $\mathrm{P}(\varepsilon \rightarrow 0)$. Denote

$$
\begin{aligned}
& \mathcal{E}_{\mu}(u, \varphi, \psi ; 0)=\frac{1}{2} \int_{0}^{T} \int_{\Omega} \frac{\partial u_{z}}{\partial r} \frac{\partial \varphi_{z}}{\partial r} \psi(t) r d r d z d t, \\
& \mathcal{E}_{\mathrm{el}}(\eta, s, \varphi, \psi ; 0)=R \int_{0}^{T} \int_{0}^{L}\left\{G_{0} \frac{\partial \eta}{\partial z} \frac{\partial \varphi_{r}}{\partial z}+\frac{E_{0}}{1-\sigma^{2}}\left(\frac{\sigma}{R} \frac{\partial s}{\partial z}+\frac{\eta}{R^{2}}\right) \varphi_{r}\right. \\
& \left.+\frac{E_{0}}{1-\sigma^{2}}\left(\frac{\partial s}{\partial z} \frac{\partial \varphi_{z}}{\partial z}-\frac{\sigma}{R} \frac{\partial \eta}{\partial z} \varphi_{z}\right)\right\} \psi(t) d z d t
\end{aligned}
$$

and

$$
\mathcal{E}_{\mathrm{src}}(A, \varphi, \psi)=\int_{0}^{T} \int_{0}^{R} A(t) \varphi_{z} \psi(t) r d r d t
$$

Definition 6 (weak formulation of the limiting problem $\mathrm{P}(\varepsilon \rightarrow 0)$ ). Let $G_{0}>0$. Vector function $\left(u_{z}, \eta, s\right) \in L^{2}(0, T ; W) \times L^{\infty}\left(0, T ; H^{1}(0, L)\right)^{2}$ such that $\left\{\partial_{t} \eta, \partial_{t} s\right\} \in L^{2}\left(0, T ; H^{-1}(0, L)\right) \times$ $L^{2}((0, T) \times(0, L))$ is called a weak solution of the limiting problem $\mathrm{P}(\varepsilon \rightarrow 0)$ if

$$
\mathcal{E}_{\mu}(u, \varphi, \psi ; 0)+\mathcal{E}_{\mathrm{el}}(\eta, s, \varphi, \psi ; 0)=-\mathcal{E}_{\mathrm{src}}(A, \varphi, \psi), \quad \text { in } \mathcal{D}^{\prime}\left(\mathbb{R}_{+}\right) \forall \varphi \in V
$$

and conditions (6.10)-(6.13) are satisfied.

If $G_{0}=0$, then we need $\eta \in L^{\infty}\left(0, T ; L^{2}(0, L)\right)$ and $\partial_{t} \eta \in L^{2}\left(0, T ; H^{-1}(0, L)\right)$.

It will be convenient to work with the weak formulation written in terms of the pressure and velocity. For this purpose we define the following.

Definition 7 (weak formulation of $\mathrm{P}(\varepsilon \rightarrow 0)$ in the pressure-velocity form). Let $G_{0}>0$. Vector function $\left\{u_{z}, \eta, s\right\} \in L^{2}(0, T ; W) \times L^{\infty}\left(0, T ; H^{1}(0, L)\right)^{2},\left\{\partial_{t} \eta, \partial_{t} s\right\} \in L^{2}\left(0, T ; H^{-1}(0, L)\right) \times$ $L^{2}((0, T) \times(0, L))$, and $p \in L^{2}((0, T) \times \Omega)$ form a weak solution of the limiting problem $\mathrm{P}(\varepsilon \rightarrow 0)$ if

$$
\begin{gathered}
\mathcal{E}_{\mu}(u, \varphi, \psi ; 0)-\int_{0}^{T} \int_{\Omega} p(\operatorname{div} \varphi) \psi r d r d z d t \\
+\mathcal{E}_{\mathrm{el}}(\eta, s, \varphi, \psi ; 0)=-\mathcal{E}_{\mathrm{src}}(A, \varphi, \psi) \quad \forall \varphi \in \mathcal{D}\left(\mathbb{R}_{+}\right) \text {and } \forall \varphi \in V_{\operatorname{div} \neq 0},
\end{gathered}
$$


and the conditions (6.10)-(6.13) are satisfied. $V_{\text {div } \neq 0}$ is defined by (4.9).

If $G_{0}=0$, then we require $\eta \in L^{\infty}\left(0, T ; L^{2}(0, L)\right)$ and $\partial_{t} \eta \in L^{2}\left(0, T ; H^{-1}(0, L)\right)$.

The following theorem shows that solutions of problems $\mathrm{P}(\varepsilon)$ converge, as $\varepsilon \rightarrow 0$, to the solution of the limiting problem.

Theorem 6.3. Any cluster point $\left\{u_{z}, p, \eta, s\right\} \in L^{2}(0, T ; W) \times L^{2}((0, T) \times \Omega) \times L^{\infty}\left(0, T ; L^{2}(0, L)\right)$ $\times L^{\infty}\left(0, T ; H^{1}(0, L)\right)$ satisfies the weak formulation of the limiting problem $\mathrm{P}(\varepsilon \rightarrow 0)$.

Proof. Consider the test functions $\varphi \in V_{\text {div } \neq 0}$ which are not divergence-free. First observe that since

$$
\frac{\mu}{\varepsilon^{2}} \frac{\partial v(\varepsilon)_{z}}{\partial r} \rightarrow \frac{\partial u_{z}}{\partial r} \quad \text { weakly in } \quad L^{2}((0, T) \times \Omega)
$$

we have

$$
\lim _{\varepsilon \rightarrow 0} \mathcal{E}_{\mu}(v(\varepsilon), \varphi, \psi ; \varepsilon)=\int_{0}^{T} \int_{\Omega} \frac{\partial u_{z}}{\partial r} \frac{\partial \varphi_{z}}{\partial r} \psi(t) r d r d z d t
$$

Next, weak convergence of $p(\varepsilon)$ gives

$$
\lim _{\varepsilon \rightarrow 0} \int_{0}^{T} \int_{\Omega} p(\varepsilon)\left(\frac{\partial \varphi_{z}}{\partial z}+\frac{\partial \varphi_{r}}{\partial r}+\frac{\varphi_{r}}{r}\right) \psi(t) r d r d z d t=\int_{0}^{T} \int_{\Omega} p(\operatorname{div} \varphi) \psi r d r d z d t .
$$

Similarly, using $\rho_{w} R h(\varepsilon) \varepsilon^{3} / \mu^{2} \leq C \varepsilon^{3 / 2}$, we get

$$
\begin{gathered}
\lim _{\varepsilon \rightarrow 0} \mathcal{E}_{\mathrm{el}}\left(\eta^{\varepsilon}, s^{\varepsilon}, \varphi, \psi ; \varepsilon\right)=R \int_{0}^{T} \int_{0}^{L}\left\{G_{0} \frac{\partial \eta}{\partial z} \frac{\partial \varphi_{r}}{\partial z}+\frac{E_{0}}{1-\sigma^{2}}\left(\frac{\sigma}{R} \frac{\partial s}{\partial z}+\frac{\eta}{R^{2}}\right) \varphi_{r}\right. \\
\left.+\frac{E_{0}}{1-\sigma^{2}}\left(\frac{\partial s}{\partial z} \frac{\partial \varphi_{z}}{\partial z}-\frac{\sigma}{R} \frac{\partial \eta}{\partial z} \varphi_{z}\right)\right\} \psi(t) d z d t
\end{gathered}
$$

Therefore, $\left\{u_{z}, p, \eta, s\right\}$ satisfies the weak form (6.15).

The proof of Theorem 6.3 implies that any cluster point $\left\{u_{z}, p, \eta, s\right\}$ satisfies the momentum equation

$$
\frac{\partial}{r \partial r}\left(r \frac{\partial u_{z}}{\partial r}\right)-\frac{\partial p}{\partial z}=0 \quad \text { in } \quad \Omega \times(0, T)
$$

the incompressibility condition (6.8), the initial and boundary conditions (6.10)-(6.13), and the following lateral boundary conditions:

$$
\begin{gathered}
p(z, t)=\frac{E_{0}}{R\left(1-\sigma^{2}\right)}\left(\sigma \frac{\partial s}{\partial z}+\frac{\eta}{R}\right)-G_{0} \frac{\partial^{2} \eta}{\partial z^{2}} \quad \text { on } \quad(0, L) \times(0, T), \\
\left.\frac{\partial u_{z}}{\partial r}\right|_{r=R}=\frac{E_{0}}{1-\sigma^{2}} \frac{\partial}{\partial z}\left(\frac{\partial s}{\partial z}+\frac{\sigma \eta}{R}\right) \quad \text { on } \quad(0, L) \times(0, T) .
\end{gathered}
$$

We note that system (6.8), (6.17), with the lateral boundary conditions (6.10), (6.18), and (6.19) and the inlet and outlet boundary conditions (6.11) and (6.12) has a unique solution. This solution coincides with the solution of the reduced problem obtained using asymptotic 
reduction, presented in section 5.3. Its weak form is the problem (6.15). Therefore, we have shown the following.

Corollary 6.4. Every convergent subsequence of solutions of the rescaled problem $\mathrm{P}(\varepsilon)$ converges weakly, as $\epsilon \rightarrow 0$, to the unique solution of the reduced problem obtained using asymptotic reduction, presented in section 5.3. The weak formulation of the reduced problem is given by (6.10)-(6.13) and (6.15).

The methods used in this section are standard and can be found in $[5,7,14,15]$. We mention that the same convergence techniques can be used in the nonlinear case when monotone operators are considered (see, e.g., $[18,2,17]$ ). Establishing the precise error estimates is nonstandard, and we present the analysis in the next section.

7. Error estimates. In this section we establish the accuracy of the approximation and the relationship between the function $v_{r}$ and the radial velocity. The literature on higher order approximations for fluid-structure problems in thin domains is sparse because of the various difficulties associated with the boundary conditions that can be prescribed on a finite domain. The closest result on higher order approximations for the Stokes flow can be found in [19], where Stokes flow through fixed domains with small thickness was studied with the prescribed velocity field as the lateral boundary condition. This is different from our approach.

There are various difficulties associated with error estimates for the fluid-structure interaction problem considered in the current paper. The main source of difficulties lies in the choice of the appropriate outlet boundary conditions. The minimum requirement for the outlet boundary conditions is that they should lead to a well-posed reduced problem. We found, for example, that periodic boundary conditions do not lead to a well-posed reduced problem. A further requirement is that the outlet boundary conditions for the reduced problem should be compatible with the fluid-structure interaction in the rest of the domain. Many "standard" boundary conditions typically used in fixed domains (rigid wall tubes) do not seem to satisfy this requirement. For example, zero tangential velocity at the outlet boundary, considered in our paper, although it gives rise to a well-posed initial-boundary-value problem, is incompatible with the flow generated in the rest of the domain. This generates a boundary layer at the outlet boundary and provides technical difficulties in obtaining the error estimates.

To get around this difficulty we construct a boundary layer in a manner similar to the one used in [8]. The construction is based on the following approach. We consider the same differential operator which holds for the original problem, but defined on a fixed domain, with the appropriate lateral boundary condition which will serve as a correction for the boundary layer effects. The rest of the boundary data is designed so that the boundary layer solution decreases exponentially away from the outlet boundary and therefore does not contaminate the original solution to the leading order accuracy. Nevertheless, due to the fact that the boundary layer solution ignores the interaction between the wall and the fluid, important shear stress effects will be created at the lateral boundary. This will give rise to a lower accuracy in the error estimate. More precisely, we will get an estimate of order $\mathcal{O}\left(\varepsilon^{3 / 2}\right)$. This is by $\mathcal{O}(\sqrt{\varepsilon})$ weaker then the analogous estimates in [19]. Getting a better estimate would, in turn, lead to a complicated modification of the effective equations.

We construct the boundary layer explicitly by considering the following abstract problem on a semi-infinite rigid-wall cylinder $Z^{-}=S \times \mathbb{R}_{-}$, where $S=\{r<R\} \times\left\{y_{3}=0\right\}$ : 


$$
\begin{gathered}
-\Delta \beta^{b l}+\nabla \pi^{b l}=0 \quad \text { in } \quad Z^{-}, \\
\operatorname{div} \beta^{b l}=0 \quad \text { in } \quad Z^{-}, \\
\beta_{r}^{b l}=v_{r}(t, r, L) \quad \text { on } \quad y_{3}=0, \\
-2 \frac{\partial \beta_{z}^{b l}}{\partial y_{3}}+\pi^{b l}=-2 \frac{\partial v_{z}}{\partial z}(t, r, L) \quad \text { on } \quad y_{3}=0, \\
\beta^{b l}=0 \quad \text { on } \quad \partial S \times \mathbb{R}_{-} .
\end{gathered}
$$

Proposition 7.11 from [8] provides the existence of a unique variational solution $\left\{\beta^{b l}, \pi^{b l}\right\} \in$ $C_{l o c}^{\infty}\left(Z^{+} \cup Z^{-}\right)^{3} \times C_{l o c}^{\infty}\left(Z^{+} \cup Z^{-}\right)$for problem (7.1)-(7.5) such that there exist constants $\gamma_{0}>0$ and $C_{\pi}$ satisfying

$$
\left\{\begin{array}{l}
e^{\gamma_{0}\left|y_{3}\right|} D^{\alpha} \beta^{b l} \in L^{2}\left(Z^{-}\right)^{9} \quad \text { for each multi-index } \alpha \in \mathbb{N}^{3} \text { and } \alpha=0 \\
e^{\gamma_{0}\left|y_{3}\right|}\left(\pi^{b l}-C_{\pi}\right) \in L^{2}\left(Z^{-}\right), \quad \text { and } \\
e^{\gamma_{0}\left|y_{3}\right|} D^{\alpha} \pi^{b l} \in L^{2}\left(Z^{-}\right)^{9} \quad \text { for each multi-index } \alpha \in \mathbb{N}^{3}
\end{array}\right.
$$

In addition, $\int_{S} \beta_{z}^{b l}=0$ and we have $W^{2, q}$-regularity of $\beta^{b l}$ and $W^{1, q}$-regularity of $\pi^{b l}$ at $\partial S \times\{0\}$.

Define the boundary layer velocity and the boundary layer pressure to be

$$
\beta^{\varepsilon}(t, r, z)=\varepsilon \beta^{b l}\left(t, r, \frac{z-L}{\varepsilon}\right), \quad \pi^{\varepsilon}(t, r, z)=\varepsilon^{2}\left(\pi^{b l}\left(t, r, \frac{z-L}{\varepsilon}\right)-C_{\pi}\right) .
$$

The boundary layer velocity and pressure satisfy

$$
\begin{gathered}
\operatorname{Div}_{\varepsilon}\left(\pi^{\varepsilon} I-2 \varepsilon^{2} D_{\varepsilon}\left(\beta^{\varepsilon}\right)\right)=0 \quad \text { in } \Omega \times \mathbb{R}_{+}, \\
\operatorname{div}_{\varepsilon} \beta^{\varepsilon}=0 \quad \text { in } \Omega \times \mathbb{R}_{+}, \\
\beta_{r}^{\varepsilon}=\varepsilon v_{r} \text { and } \pi^{\varepsilon}-2 \varepsilon^{2} \frac{\partial \beta_{z}^{\varepsilon}}{\partial z}=\varepsilon^{2}\left(-2 \frac{\partial v_{z}}{\partial z}-C_{\pi}\right) \quad \text { for } z=L,
\end{gathered}
$$

and the following estimates for the behavior at the lateral and at the inlet boundary:

$$
\begin{gathered}
\left\|\left.\pi^{\varepsilon}\right|_{r=R}-\left.2 \varepsilon \frac{\partial \beta_{r}^{\varepsilon}}{\partial r}\right|_{r=R}\right\|_{L^{2}(0, L)} \leq C \varepsilon^{5 / 2} \quad \forall t>0, \\
\left\|\left.\varepsilon^{2}\left(\frac{\partial \beta_{r}^{\varepsilon}}{\partial z}+\frac{1}{\varepsilon} \frac{\partial \beta_{z}^{\varepsilon}}{\partial r}\right)\right|_{r=R}\right\|_{L^{2}(0, L)} \leq C \varepsilon^{5 / 2} \quad \forall t>0, \\
\beta_{r}^{\varepsilon} \text { and } \pi^{\varepsilon}-2 \varepsilon \frac{\partial \beta_{z}^{\varepsilon}}{\partial z} \text { are exponentially small for } z=0 .
\end{gathered}
$$

Furthermore, the following estimate holds on domain $\Omega$ :

$$
\varepsilon\left\|\beta^{\varepsilon}\right\|_{L^{2}(\Omega)^{3}}+\left\|\pi^{\varepsilon}\right\|_{L^{2}(\Omega)}+\varepsilon\left\|\frac{\partial \beta^{\varepsilon}}{\partial r}\right\|_{L^{2}(\Omega)^{3}}+\left\|\frac{\partial \pi^{\varepsilon}}{\partial r}\right\|_{L^{2}(\Omega)} \leq C \varepsilon^{5 / 2} .
$$


We are now in a position to estimate the difference between the solution of the rescaled problem and the solution of the limiting problem, modified by the boundary layer. We introduce the following notation:

$$
\begin{gathered}
\bar{p}(\varepsilon)=p(\varepsilon)-p+\pi^{\varepsilon}, \bar{v}(\varepsilon)=\frac{\mu}{\varepsilon^{2}} v(\varepsilon)-v_{z} \vec{e}_{z}-\varepsilon v_{r} \vec{e}_{r}+\beta^{\varepsilon}, \\
\bar{s}(\varepsilon)=s^{\varepsilon}-s, \bar{\eta}(\varepsilon)=\eta^{\varepsilon}-\varepsilon \eta .
\end{gathered}
$$

The following theorem provides the error estimates.

Theorem 7.1. Suppose that $A \in C_{0}^{\infty}(0,+\infty)$, and let the parameters satisfy

$$
\left|E_{0}-\frac{h(\varepsilon) E(\varepsilon)}{\varepsilon}\right| \leq C \varepsilon^{3 / 2},\left|G_{0}-h(\varepsilon) G(\varepsilon) k(\varepsilon) \varepsilon\right| \leq C \varepsilon^{3 / 2}, \frac{\rho_{w} h(\varepsilon) \varepsilon^{3}}{\mu^{2}} \leq C \varepsilon^{3 / 2} .
$$

Then for each fixed $\epsilon>0$ the following estimates hold for (1) the error in the velocity and pressure:

$$
\begin{gathered}
\left\|\frac{\bar{v}(\varepsilon)_{r}}{r}\right\|_{L^{2}}+\left\|\bar{v}(\varepsilon)_{z}\right\|_{L^{2}}+\left\|\frac{\partial \bar{v}(\varepsilon)_{r}}{\partial r}\right\|_{L^{2}}+\left\|\frac{\partial \bar{v}(\varepsilon)_{z}}{\partial r}\right\|_{L^{2}} \leq C \varepsilon^{3 / 2}\|A\|_{H^{5}(0, T)}, \\
\left\|\frac{\partial \bar{v}(\varepsilon)_{r}}{\partial z}\right\|_{L^{2}}+\left\|\frac{\partial \bar{v}(\varepsilon)_{z}}{\partial z}\right\|_{L^{2}} \leq C \varepsilon^{1 / 2}\|A\|_{H^{5}(0, T)} \\
\|\bar{p}(\varepsilon)\|_{L^{2}} \leq C \varepsilon^{3 / 2}\|A\|_{H^{5}(0, T)}
\end{gathered}
$$

where the $L^{2}$-norms are defined on $\Omega \times(0, T)$, and for (2) the error in the radial and longitudinal displacement:

$$
\begin{aligned}
& \sup _{0 \leq t \leq T}\left\{\sqrt{G_{0}}\left\|\frac{\partial \bar{\eta}(\varepsilon)}{\varepsilon \partial z}(t)\right\|_{L^{2}}+\sqrt{E_{0}}\left\|\frac{\bar{\eta}(\varepsilon)}{\varepsilon}(t)\right\|_{L^{2}}+\left\|\frac{\partial \bar{s}(\varepsilon)}{\partial z}(t)\right\|_{L^{2}}\right\} \\
& \leq C \varepsilon^{3 / 2}\|A\|_{H^{5}(0, T)},
\end{aligned}
$$

where the $L^{2}$-norms are defined on $(0, L)$.

The proof of this theorem is rather technical, and we enclose it in the appendix. Here we summarize the main steps.

We first calculate the PDE and the boundary conditions that are satisfied by the error $(\bar{v}, \bar{p}, \bar{\eta}, \bar{s})$. Without loss of generality suppose $G_{0}>0$. Using the equations for the rescaled problem (4.1)-(4.6), the reduced equations obtained via asymptotic reduction (5.3), (5.4)-(5.5), (5.9), and (5.10), and the boundary layer problem (7.8)-(7.14), we obtain the PDEs that hold in $\Omega \times \mathbb{R}_{+}$:

$$
\begin{gathered}
\operatorname{Div}_{\varepsilon}\left(\bar{p}(\varepsilon) I-2 \varepsilon^{2} D_{\varepsilon}(\bar{v}(\varepsilon))\right)=\varepsilon^{2}\left(2 \frac{\partial^{2} v_{z}}{\partial z^{2}} \vec{e}_{z}+\varepsilon \frac{\partial^{2} v_{r}}{\partial z^{2}} \vec{e}_{r}\right), \\
\operatorname{div}_{\varepsilon} \bar{v}(\varepsilon)=0 .
\end{gathered}
$$

At the lateral boundary $\{R\} \times(0, L) \times \mathbb{R}_{+}$the error satisfies

$$
\begin{aligned}
\bar{p}(\varepsilon)-2 \varepsilon \frac{\partial \bar{v}(\varepsilon)_{r}}{\partial r} & =F_{r}(\varepsilon)+\Phi_{r}^{\varepsilon}, \\
-\varepsilon^{2}\left(\frac{\partial \bar{v}(\varepsilon)_{r}}{\partial z}+\frac{1}{\varepsilon} \frac{\partial \bar{v}(\varepsilon)_{z}}{\partial r}\right) & =F_{z}(\varepsilon)+\Phi_{z}^{\varepsilon},
\end{aligned}
$$


where $F_{r}(\varepsilon)$ and $F_{z}(\varepsilon)$ are the elastic forces generated by the error on the membrane, given by

$$
\begin{aligned}
F_{r}(\varepsilon) & =\frac{h(\varepsilon) E(\varepsilon)}{\varepsilon} \frac{1}{R\left(1-\sigma^{2}\right)}\left(\sigma \frac{\partial \bar{s}(\varepsilon)}{\partial z}+\frac{\bar{\eta}(\varepsilon)}{R \varepsilon}\right)+\left(\omega^{\varepsilon}\right)^{2} \rho_{w} h(\varepsilon) \frac{\partial^{2} \bar{\eta}(\varepsilon)}{\partial t^{2}} \\
& -h(\varepsilon) G(\varepsilon) k(\varepsilon) \varepsilon \frac{\partial^{2}}{\partial z^{2}} \frac{\bar{\eta}(\varepsilon)}{\varepsilon} \\
F_{z}(\varepsilon) & =-h(\varepsilon) E(\varepsilon) \frac{1}{1-\sigma^{2}} \frac{\partial}{\partial z}\left(\frac{\partial \bar{s}(\varepsilon)}{\partial z}+\frac{\sigma \bar{\eta}(\varepsilon)}{R \varepsilon}\right)+\left(\omega^{\varepsilon}\right)^{2} \rho_{w} h(\varepsilon) \frac{\partial^{2}}{\partial t^{2}} \bar{s}(\varepsilon),
\end{aligned}
$$

and $\Phi_{r}^{\varepsilon}$ and $\Phi_{z}^{\varepsilon}$ are the contributions of the remaining terms, given by

$$
\begin{aligned}
\Phi_{r}^{\varepsilon} & =-\frac{\partial}{\partial z} \Phi_{r}^{1, \varepsilon}+\Phi_{r}^{2, \varepsilon}, \\
\Phi_{z}^{\varepsilon} & =-\frac{\partial}{\partial z} \Phi_{z}^{1, \varepsilon}+\Phi_{z}^{2, \varepsilon},
\end{aligned}
$$

where

$$
\begin{aligned}
\Phi_{r}^{1, \varepsilon} & =\varepsilon^{2}\left(\frac{R^{2}}{8} \frac{\partial p}{\partial z}+\frac{\partial s}{\partial t}\right)\left(\frac{\partial v_{z}}{\partial z}+2 \frac{\partial v_{r}}{\partial r}\right)-\left(G_{0}-h(\varepsilon) G(\varepsilon) k(\varepsilon) \varepsilon\right) \frac{\partial \eta}{\partial z} \\
\Phi_{r}^{2, \varepsilon} & =\left(\omega^{\varepsilon}\right)^{2} \varepsilon \rho_{w} h(\varepsilon) \frac{\partial^{2} \eta}{\partial t^{2}}+\pi^{\varepsilon}-2 \varepsilon \frac{\partial \beta_{r}^{\varepsilon}}{\partial r}-\left(E_{0}-\frac{h(\varepsilon) E(\varepsilon)}{\varepsilon}\right) \frac{\sigma \frac{\partial s}{\partial z}+\frac{\eta}{R}}{R\left(1-\sigma^{2}\right)}
\end{aligned}
$$

and

$$
\begin{aligned}
\Phi_{z}^{1, \varepsilon} & =-\left(E_{0}-\frac{h(\varepsilon) E(\varepsilon)}{\varepsilon}\right) \frac{\varepsilon}{1-\sigma^{2}} \frac{\partial s}{\partial z}-\varepsilon^{3} \frac{R}{2}\left(\frac{R^{2}}{8} \frac{\partial^{2} p}{\partial z^{2}}-\frac{\partial^{2} s}{\partial t \partial z}\right), \\
\Phi_{z}^{2, \varepsilon} & =\left(\omega^{\varepsilon}\right)^{2} \rho_{w} h(\varepsilon) \frac{\partial^{2} s}{\partial t^{2}}+\varepsilon \frac{\partial \beta_{z}^{\varepsilon}}{\partial r}+\left(E_{0}-\frac{h(\varepsilon) E(\varepsilon)}{\varepsilon}\right) \frac{\varepsilon}{1-\sigma^{2}} \frac{\partial}{\partial z} \frac{\sigma \eta}{R} .
\end{aligned}
$$

Finally, at the inlet and the outlet boundaries we have

$$
\begin{gathered}
\bar{v}(\varepsilon)_{r}=0 \text { and } \bar{p}(\varepsilon)-2 \varepsilon^{2} \frac{\partial \bar{v}(\varepsilon)_{z}}{\partial z}=-\varepsilon^{2} C_{\pi} \text { for } z=L \\
\bar{v}(\varepsilon)_{r} \text { and } \bar{p}(\varepsilon)-2 \varepsilon^{2} \frac{\partial \bar{v}(\varepsilon)_{z}}{\partial z} \text { are exponentially small for } z=0 .
\end{gathered}
$$

Next we write the variational formulation of system (7.20)-(7.25) (see (8.1)) and obtain an energy equality. This is (8.2) in the appendix. From the energy equality, $L^{2}$-estimates for (7.20)-(7.25) are calculated. Estimate (7.12) is crucial in obtaining the leading order behavior of the error as $\mathcal{O}\left(\epsilon^{3 / 2}\right)$. We recall that estimate (7.12) is a consequence of the geometry of the domain and the choice of the appropriate scaling of the boundary layer. The appropriate scaling of the boundary layer is determined from the underlying operator and the time-scale of the problem. Details of the proof are presented in the appendix.

In Figure 7.1 we show numerical simulations obtained for the pressure drop $A(t)$ prescribed on the right boundary $z=L$, given by $A(t)=950 \sin (2 \pi t) \mathrm{Pa}$. The reference pressure 

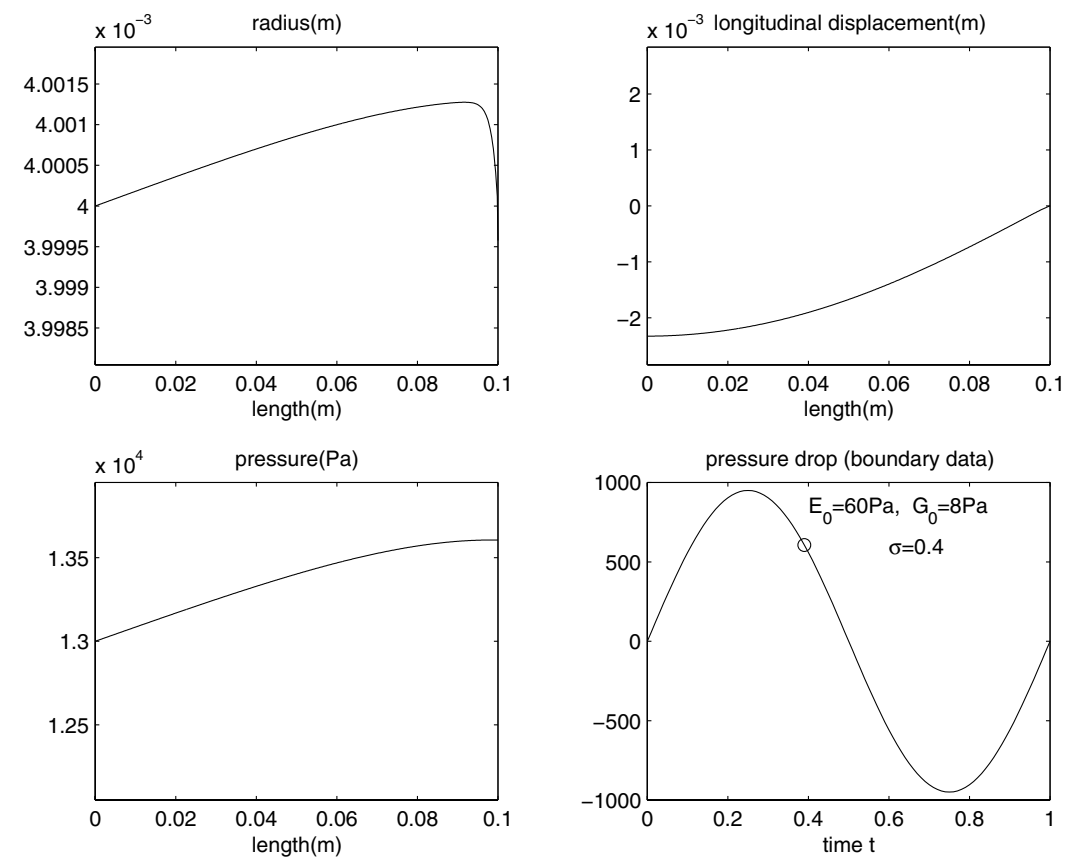

Figure 7.1. Numerical simulation: Effective displacements with the given pressure drop $A(t)$. The reference pressure is $1.3 \times 10^{4} \mathrm{~Pa}$.

$P_{0}=1.3 \times 10^{4} \mathrm{~Pa}$. The initial configuration is unperturbed, corresponding to the initial radius $R=0,0004 \mathrm{~m}$ with the initial radial and longitudinal displacement equal to zero. The values of Young's modulus and the shear modulus are $E_{0}=60 \mathrm{~Pa}, G_{0}=8 \mathrm{~Pa}$, and the Poisson ratio is $\sigma=0.4$. Movies showing the dynamics of the vessel wall can be viewed at http://www.math.uh.edu/ canic/ elisejerome/smallarteries.html. The figure below is taken at time $t=0.40$. The dot on the graph of the pressure data $A(t)$ indicates the time in the sinusoidal cycle when the "snap-shot" is taken. We see clearly the formation of the boundary layer in the radial displacement at the boundary $z=L$. The pressure wave coming from the right "pushes" the particles on the membrane to the left, introducing negative longitudinal displacement in the first half of the sinusoidal pulse. This is consistent with the continuity of velocity and forces at the lateral boundary. The radial displacement is positive, and it is decreasing as the points move to the left, away from the right boundary $z=L$. Notice how the radial displacement is by $\varepsilon$ order of magnitude smaller than the longitudinal displacement. The movie mentioned above shows how the wall particles move up and to the left in the first half of the sinusoidal cycle and down and to the right in the second half of the pressure cycle.

7.1. The reduced problem and error in physical domain. We conclude this section by writing the reduced equation in the physical (spatial) coordinates, namely, in the domain $\Omega_{\varepsilon}$. Since $p$ is independent of $r$, the equation for the pressure can be obtained directly from (5.25) by taking into account that the physical inner vessel radius equals $\varepsilon R$ and that $\varepsilon$ is equal to 
the inner vessel radius divided by the length of the vessel $L$. We obtain

$$
\left\{\begin{array}{l}
\frac{\partial}{\partial t}\left\{\left(\frac{5}{2}-2 \sigma\right) p-\left(1-\sigma^{2}\right) \frac{G_{0} L^{2}}{2 E_{0}} \frac{\partial^{2} p}{\partial z^{2}}\right\}=\frac{\partial^{2}}{\partial z^{2}}\left\{\frac{E_{0} L}{8} p-\frac{G_{0} L^{3}}{8} \frac{\partial^{2} p}{\partial z^{2}}\right\} \\
p(0, t)=0, \quad p(L, t)=A(t) \quad \text { in } \quad(0, T), \\
\frac{\partial^{2} p}{\partial z^{2}}(0, t)=0, \quad \frac{E_{0} L}{8\left(1-\sigma^{2}\right)} \frac{\partial^{2} p}{\partial z^{2}}(L, t)=\frac{1}{2} \frac{d A}{d t} \quad \text { in } \quad(0, T), \\
p(z, 0)=0 \quad \text { in } \quad(0, L) .
\end{array}\right.
$$

We emphasize that this problem is written in the $\tilde{t}$ time-scale.

The error between the solution of the reduced problem defined on $\Omega_{\varepsilon}$ and the solution of the original problem $P^{\varepsilon}$ is obtained as follows. First, recall the notation used in section 5, where the asymptotic equations were written in terms of

$$
p=p^{0}+\varepsilon p^{1}, s=s^{0}+\varepsilon s^{1}, \eta=\eta^{0}+\varepsilon \eta^{1}, v_{r}=v_{r}^{1}+\varepsilon v_{r}^{2}, \quad \text { and } \quad v_{z}=v_{z}^{0}+\varepsilon v_{z}^{1} .
$$

Then asymptotic expansions of the rescaled quantities (4.19)-(4.22) imply the following asymptotic expansions on $\Omega_{\varepsilon}$ :

$$
\begin{gathered}
v^{\varepsilon}(z, r, t)=\sum_{i \geq 0} \varepsilon^{i} v^{i}(z, r, t), \quad p^{\varepsilon}(z, r, t)=\sum_{i \geq 0} \epsilon^{i} p^{i}(z, r, t), \\
\eta^{\varepsilon}(z, t)=\varepsilon \sum_{i \geq 0} \epsilon^{i} \eta^{i}(z, t), \quad s^{\varepsilon}(z, t)=\sum_{i \geq 0} \epsilon^{i} s^{i}(z, t) .
\end{gathered}
$$

Denote the error in the velocity and pressure, modified by the boundary layer, by

$$
\begin{aligned}
& \bar{v}^{\varepsilon}=v^{\varepsilon}-v_{z}(z, r / \varepsilon, t) \vec{e}_{z}-\varepsilon v_{r}(z, r / \varepsilon, t) \vec{e}_{r}+\beta^{\varepsilon}(z, r / \varepsilon, t), \\
& \bar{p}^{\varepsilon}=p^{\varepsilon}-p(z, t)+\pi^{b l}(z, r / \varepsilon, t) .
\end{aligned}
$$

Notice that

$$
\begin{gathered}
v_{r}^{\varepsilon}-\varepsilon v_{r}=\varepsilon^{3} \sum_{i \geq 3} \varepsilon^{i-3} v^{i}, \quad v_{z}^{\varepsilon}-v_{z}=\varepsilon^{2} \sum_{i \geq 2} \varepsilon^{i-2} v^{i}, \quad p^{\varepsilon}-p=\varepsilon^{2} \sum_{i \geq 2} \varepsilon^{i-2} p^{i}, \\
\eta^{\varepsilon}-\varepsilon \eta=\varepsilon^{3} \sum_{i \geq 3} \varepsilon^{i-3} \eta^{i}, \quad s^{\varepsilon}-s=\varepsilon^{2} \sum_{i \geq 2} \varepsilon^{i-2} s^{i} .
\end{gathered}
$$

Theorem 7.1 implies the following error estimates on $\Omega_{\varepsilon} \times(0, T)$ :

$$
\begin{gathered}
\left\|\frac{\bar{v}_{r}^{\varepsilon}}{r}\right\|_{L^{2}\left(\Omega_{\varepsilon} \times(0, T)\right)}+\frac{1}{\sqrt{\left|\Omega_{\varepsilon}\right|}}\left\|\bar{v}_{z}^{\varepsilon}\right\|_{L^{2}\left(\Omega_{\varepsilon} \times(0, T)\right)}+\left\|\frac{\partial \bar{v}_{z}^{\varepsilon}}{\partial r}\right\|_{L^{2}\left(\Omega_{\varepsilon} \times(0, T)\right)} \leq C \varepsilon^{3 / 2}\|A\|_{H^{5}(0, T)}, \\
+\left\|\frac{\partial \bar{v}_{r}^{\varepsilon}}{\partial r}\right\|_{L^{2}\left(\Omega_{\varepsilon} \times(0, T)\right)} \leq C \varepsilon^{1 / 2}\|A\|_{H^{5}(0, T)}, \\
\frac{1}{\sqrt{\left|\Omega_{\varepsilon}\right|}}\left\|\frac{\partial \bar{v}_{r}^{\varepsilon}}{\partial z}\right\|_{L^{2}\left(\Omega_{\varepsilon} \times(0, T)\right)}+\frac{1}{\sqrt{\left|\Omega_{\varepsilon}\right|}}\left\|\frac{\partial \bar{v}_{z}^{\varepsilon}}{\partial z}\right\|_{L^{2}\left(\Omega_{\varepsilon} \times(0, T)\right)} \leq C \varepsilon^{3 / 2}\|A\|_{H^{5}(0, T)} . \\
\frac{1}{\sqrt{\left|\Omega_{\varepsilon}\right|}}\left\|\bar{p}^{\varepsilon}\right\|_{L^{2}\left(\Omega_{\varepsilon} \times(0, T)\right)} \leq C \varepsilon^{2}
\end{gathered}
$$


The estimates for the displacement (7.19) remain unchanged.

Finally we mention that if we assume that the approximation of the data in (7.15) is of order $\varepsilon^{2}$, the resulting error estimates are of order $\varepsilon^{2}$ in the norms defined on the domain $\Omega_{\varepsilon}$ excluding the outlet boundary. Furthermore, we can obtain local estimates of order $\varepsilon^{2}$ for the derivatives with respect to $z$ of all orders.

8. Appendix. Here we present the proof of Theorem 7.1.

Proof. We start by writing the variational form of system (7.20)-(7.25) satisfied by the error $(\bar{v}, \bar{p}, \bar{\eta}, \bar{s})$ :

$$
\begin{gathered}
\mathcal{E}_{\mu}\left(\bar{v},\left(\varphi_{r} / \varepsilon, \varphi_{z}\right), \psi ; \varepsilon\right)+\mathcal{E}_{\mathrm{el}}\left(\bar{\eta}, \bar{s},\left(\varphi_{r} / \varepsilon, \varphi_{z}\right), \psi ; \varepsilon\right) \\
=\varepsilon^{2} \int_{0}^{t} \int_{\Omega}\left(2 \frac{\partial^{2} v_{z}}{\partial z^{2}} \varphi_{z}+\varepsilon \frac{\partial^{2} v_{r}}{\partial z^{2}} \varphi_{r}\right) \psi(\tau) r d r d z d \tau \\
-\frac{R}{\varepsilon} \int_{0}^{t} \int_{0}^{L}\left\{\Phi_{r}^{2, \varepsilon} \varphi_{r}(t, R, z)+\Phi_{z}^{2, \varepsilon} \varphi_{z}(t, R, z)+\Phi_{r}^{1, \varepsilon} \frac{\partial \varphi_{r}}{\partial z}+\Phi_{z}^{1, \varepsilon} \frac{\partial \varphi_{z}}{\partial z}\right\} \psi(\tau) d z d \tau \psi(\tau) r d r d \tau, \\
(8.1) \quad-\varepsilon^{2} \int_{0}^{t} \int_{0}^{R} C_{\pi} \varphi_{z}(r, L) \psi(\tau) r d r d \tau \text { in } \mathcal{D}^{\prime}\left(\mathbb{R}_{+}\right) \forall \varphi \in V_{d i v \neq 0}, \operatorname{div}_{\varepsilon} \varphi=0 .
\end{gathered}
$$

Consider exponentially small quantities to be equal to zero. Then $\bar{v}(\varepsilon) \in V$ for every $t$ and $\bar{v}(\varepsilon)$ can be used as a test function to obtain the following energy equality:

$$
\begin{aligned}
& 2 \int_{0}^{t} \int_{\Omega}\left(\left(\frac{\partial \bar{v}(\varepsilon)_{r}}{\partial r}\right)^{2}+\left(\frac{\bar{v}(\varepsilon)_{r}}{\partial r}\right)^{2}+\varepsilon^{2}\left(\frac{\partial \bar{v}(\varepsilon)_{z}}{\partial z}\right)^{2}\right)+\int_{0}^{t} \int_{\Omega}\left(\varepsilon \frac{\partial \bar{v}(\varepsilon)_{r}}{\partial z}+\frac{\partial \bar{v}(\varepsilon)_{z}}{\partial r}\right)^{2} \\
& +\frac{R}{2}\left\{\frac{\left(\omega^{\varepsilon}\right)^{2} \rho_{w} h(\varepsilon)}{\varepsilon} \int_{0}^{L}\left(\left|\frac{\partial \bar{\eta}(\varepsilon)}{\partial t}(t)\right|^{2}+\left|\frac{\partial \bar{s}(\varepsilon)}{\partial t}(t)\right|^{2}\right) d z+\varepsilon h(\varepsilon) G(\varepsilon) k(\varepsilon) \int_{0}^{L}\left|\frac{\partial \bar{\eta}(\varepsilon)}{\varepsilon \partial z}(t)\right|^{2}\right. \\
& \left.+\frac{h(\varepsilon) E(\varepsilon)}{\varepsilon\left(1-\sigma^{2}\right)}\left(\sigma \int_{0}^{L}\left(\frac{\bar{\eta}(\varepsilon)}{\varepsilon R}(t)-\frac{\partial \bar{s}(\varepsilon)}{\partial z}(t)\right)^{2}+(1-\sigma) \int_{0}^{L}\left(\left|\frac{\bar{\eta}(\varepsilon)}{\varepsilon R}(t)\right|^{2}+\left|\frac{\partial \bar{s}(\varepsilon)}{\partial z}(t)\right|^{2}\right)\right)\right\} \\
& =-R \int_{0}^{L}\left(\Phi_{r}^{2, \varepsilon}(t) \frac{\bar{\eta}(\varepsilon)}{\varepsilon}(t)+\Phi_{z}^{2, \varepsilon}(t) \frac{\bar{s}(\varepsilon)}{\varepsilon}(t)+\Phi_{r}^{1, \varepsilon}(t) \frac{\partial \bar{\eta}(\varepsilon)}{\varepsilon \partial z}(t)+\Phi_{z}^{1, \varepsilon}(t) \frac{\partial \bar{s}(\varepsilon)}{\varepsilon \partial z}(t)\right) \\
& +R \int_{0}^{t} \int_{0}^{L}\left\{\frac{\partial}{\partial \tau} \Phi_{r}^{2, \varepsilon} \frac{\bar{\eta}(\varepsilon)}{\varepsilon}+\frac{\partial}{\varepsilon \partial \tau} \Phi_{z}^{2, \varepsilon} \bar{s}(\varepsilon)+\frac{\partial}{\partial \tau} \Phi_{r}^{1, \varepsilon} \frac{\partial \bar{\eta}(\varepsilon)}{\varepsilon \partial z}+\frac{\partial}{\partial \tau} \Phi_{z}^{1, \varepsilon} \frac{\partial \bar{s}(\varepsilon)}{\varepsilon \partial z}\right\} \\
& -\varepsilon^{2} \int_{0}^{t} \int_{0}^{R} C_{\pi} \bar{v}(\varepsilon)_{z}(\tau, r, L) r d r d \tau+\varepsilon^{2} \int_{0}^{t} \int_{\Omega}\left(2 \frac{\partial^{2} v_{z}}{\partial z^{2}} \bar{v}(\varepsilon)_{z}+\varepsilon \frac{\partial^{2} v_{r}}{\partial z^{2}} \bar{v}(\varepsilon)_{r}\right) r d r d z d \tau .
\end{aligned}
$$

Now hypothesis (7.15), the regularity of $p$, and estimates (7.11)-(7.12) allow us to use the Gronwall inequality to estimate all the terms involving $\Phi^{j, \varepsilon}$ by $C \varepsilon^{3 / 2}$ times the norm of $\bar{v}(\varepsilon)$ at $r=R$. We note that because of (7.12) we cannot get an estimate better than $\mathcal{O}\left(\varepsilon^{3 / 2}\right)$.

Next, the outlet term $\varepsilon^{2} \int_{0}^{t} \int_{0}^{R} C_{\pi} \bar{v}(\varepsilon)_{z}(\tau, r, L) r d r d \tau$ is estimated as in the derivation of 
the a priori estimates. Observe that

$$
\begin{gathered}
-\varepsilon^{2} \int_{0}^{t} \int_{0}^{R} C_{\pi} \bar{v}(\varepsilon)_{z}(\tau, r, L) r d r d \tau=\varepsilon^{2} \int_{0}^{t} \int_{0}^{R} \frac{C_{\pi}}{L} \bar{v}(\varepsilon)_{z}(\tau, r, z) r d r d z d \tau \\
+\frac{R \varepsilon^{2} C_{\pi}}{L} \int_{0}^{t} \int_{0}^{L} z \bar{\eta}(\varepsilon)_{z}(\tau, z) d z d \tau \\
\left|\frac{R \varepsilon^{2} C_{\pi}}{L} \int_{0}^{t} \int_{\Omega} \bar{v}(\varepsilon)_{z}(\tau, r, z) r d r d z d \tau-\frac{R^{2} \varepsilon^{2} C_{\pi}}{2 L} \int_{0}^{L} \bar{s}(\varepsilon)(t) d z\right| \\
\leq \frac{R^{2} \varepsilon^{2}\left|C_{\pi}\right|}{2 \sqrt{L}}\left\|\varepsilon D_{\varepsilon}(\bar{v}(\varepsilon))\right\|_{L^{2}((0, t) \times \Omega)} .
\end{gathered}
$$

Therefore, the outlet term is controlled by the sum of two terms - a lateral boundary term, which could be included in $\Phi_{r}^{2, \varepsilon}$, and a term estimated by $C \varepsilon^{2}\left\|\varepsilon D_{\varepsilon}(\bar{v}(\varepsilon))\right\|_{L^{2}((0, t) \times \Omega)}$.

The volume term is more difficult to deal with since we have to use the $L^{2}$-norm of the symmetrized gradient. Rewrite the volume term in two parts:

$$
I_{1}=\varepsilon^{2} \int_{0}^{t} \int_{\Omega} 2 \frac{\partial^{2} v_{z}}{\partial z^{2}}(\tau, r, z) \bar{v}(\varepsilon)_{z}(\tau, r, z) r d r d z d \tau
$$

and

$$
I_{2}=\varepsilon^{3} \int_{0}^{t} \int_{\Omega} \frac{\partial^{2} v_{r}}{\partial z^{2}}(\tau, r, z) \bar{v}(\varepsilon)_{r}(\tau, r, z) r d r d z d \tau
$$

Furthermore, note that $\int_{0}^{R} 2 \xi \frac{\partial^{2} v_{z}}{\partial z^{2}}(\tau, \xi, z) d \xi=-2 R \frac{\partial^{2} \eta}{\partial t \partial z}$, and get

$$
\begin{aligned}
I_{1} & =-2 \varepsilon^{2} \int_{0}^{t} \int_{0}^{L} R \frac{\partial^{2} \eta}{\partial t \partial z} \frac{\partial \bar{s}(\varepsilon)}{\partial \tau}-2 \varepsilon^{2} \int_{0}^{t} \int_{\Omega}\left(\frac{1}{r} \int_{0}^{r} \xi \frac{\partial^{2} v_{z}}{\partial z^{2}} d \xi\right) \frac{\partial \bar{v}(\varepsilon)_{z}}{\partial r} r d r d z d \tau \\
& =-2 \varepsilon^{2} \int_{0}^{t} \int_{0}^{L} R \frac{\partial^{2} \eta}{\partial t \partial z} \frac{\partial \bar{s}(\varepsilon)}{\partial \tau}-2 \varepsilon^{2} \int_{0}^{t} \int_{\Omega}\left(\frac{1}{r} \int_{0}^{r} \xi \frac{\partial^{2} v_{z}}{\partial z^{2}} d \xi\right)\left(\frac{\partial \bar{v}(\varepsilon)_{z}}{\partial r}+\varepsilon \frac{\partial \bar{v}(\varepsilon)_{r}}{\partial z}\right) \\
& +2 \varepsilon^{3} \int_{0}^{t} \int_{\Omega} \frac{\partial}{\partial z}\left(\frac{1}{r} \int_{0}^{r} \xi \frac{\partial^{2} v_{z}}{\partial z^{2}} d \xi\right) \bar{v}(\varepsilon)_{r} r d r d z d \tau
\end{aligned}
$$

Therefore, $I_{1}$ is a sum of three terms. The first is controlled in the same way as the terms involving $\Phi^{j, \varepsilon}$, the second is easily estimated using the $L^{2}$-norm of the symmetrized gradient, and the third is to be estimated as a part of

$$
I_{3}=I_{2}+2 \varepsilon^{3} \int_{0}^{t} \int_{\Omega} \frac{\partial}{\partial z}\left(\frac{1}{r} \int_{0}^{r} \xi \frac{\partial^{2} v_{z}}{\partial z^{2}} d \xi\right) \bar{v}(\varepsilon)_{r} r d r d z d \tau .
$$

Since $v_{r}+\frac{2}{r} \int_{0}^{r} \xi \frac{\partial v_{z}}{\partial z} d \xi=-v_{r}$, we have

$$
I_{3}=-\varepsilon^{3} \int_{0}^{t} \int_{\Omega} \frac{\partial^{2} v_{r}}{\partial z^{2}} \bar{v}(\varepsilon)_{r} r d r d z d \tau=\varepsilon^{3} \int_{0}^{t} \int_{\Omega} r \frac{\partial^{2} v_{r}}{\partial z^{2}}\left(\frac{\partial \bar{v}(\varepsilon)_{r}}{\partial r}+\varepsilon \frac{\partial \bar{v}(\varepsilon)_{z}}{\partial z}\right) r d r d z d \tau
$$


and

$$
\left|I_{3}\right| \leq C \varepsilon^{3}\left(\left\|\frac{\partial \bar{v}(\varepsilon)_{r}}{\partial r}\right\|_{L^{2}((0, t) \times \Omega)}+\varepsilon\left\|\frac{\partial \bar{v}(\varepsilon)_{z}}{\partial z}\right\|_{L^{2}((0, t) \times \Omega)}\right) .
$$

Consequently, (7.19) follows and

$$
\varepsilon^{2} \int_{0}^{T}\left\|D_{\varepsilon}(\bar{v}(\varepsilon))(t)\right\|_{L^{2}(\Omega)}^{2} d t \leq C \varepsilon^{3}\|A\|^{2} .
$$

It remains to estimate the term $\left(D_{\varepsilon}(\bar{v}(\varepsilon))_{r z}\right.$. Arguing as in the proof of Proposition 3.8, we obtain

$$
\left\|\frac{\partial \bar{v}(\varepsilon)_{z}}{\partial r}\right\|_{L^{2}((0, T) \times \Omega)}+\varepsilon\left\|\frac{\partial \bar{v}(\varepsilon)_{r}}{\partial z}\right\|_{L^{2}((0, T) \times \Omega)} \leq C \varepsilon^{3 / 2}\|A\|+C_{1}\left\|\partial_{t} \bar{s}(\varepsilon)\right\|_{L^{2}((0, T) \times(0, L))} .
$$

The pressure estimate (7.18) is proved in the same way as in Proposition 4.4.

Remark 4. As in Corollary 3.9, we see that estimates (7.16) and (7.17) are also valid for the time derivative of $\bar{v}(\varepsilon)$.

Acknowledgments. The authors would like to thank Daniele Lamponi for the discussions about the model and Elise Delavaud and Jérôme Coulon for the numerical simulations shown in Figure 7.1.

\section{REFERENCES}

[1] M.A. Biot, Theory of propagation of elastic waves in a fluid-saturated porous solid. I. Lower frequency range and II. Higher frequency range, J. Acoust Soc. Amer., 28 (1956), pp. 168-191.

[2] A. Bourgeat, A. Mikelić, And R. TApiéro, Dérivation des équations moyennées décrivant un écoulement non-newtonien dans un domaine de faible épaisseur, C. R. Acad. Sci. Paris Sér. I Math., 316 (1993), pp. 965-970.

[3] S. CANIĆ, Blood flow through compliant vessels after endovascular repair: Wall deformations induced by the discontinuous wall properties, Comput. Vis. Sci., 4 (2002), pp. 147-155.

[4] S. ČANIĆ AND E.-H. KIM, Mathematical analysis of the quasilinear effects in a hyperbolic model of blood flow through compliant axi-symmetric vessels, Math. Methods Appl. Sci., to appear.

[5] P.G. Ciarlet, Plates and Junctions in Elastic Multi-structures, Rech. Math. Appl. 14, Masson, Paris, 1990.

[6] U. DinAR, Cardiovascular Fluid Dynamics, CRC Press, Boca Raton, FL, 1981.

[7] H. DRIDI, Comportement asymptotique des équations de Navier-Stokes dans des domaines "applatis," Bull. Sci. Math. (2), 106 (1982), pp. 369-385.

[8] W. JäGER AND A. Mikelić, On the effective equations for a viscous incompressible fluid flow through a filter of finite thickness, Comm. Pure Appl. Math., 51 (1998), pp. 1073-1121.

[9] G. FicherA, Existence theorems in elasticity, in Handbook der Physik VIa/2, Springer-Verlag, Berlin, 1972.

[10] L. Formaggia, F. Nobile, and A. Quarteroni, A one dimensional model for blood flow: Application to vascular prosthesis, in Mathematical Modeling and Numerical Simulation in Continuum Mechanics, Lect. Notes Comput. Sci. Engrg. 19, I. Babuska, T. Miyoshi, and P.G. Ciarlet, eds., Springer-Verlag, Berlin, 2002, pp. 137-153.

[11] Y.C. Fung, Biomechanics: Mechanical Properties of Living Tissues, Springer-Verlag, New York, 1993.

[12] J. Keener And J. Sneyd, Mathematical Physiology, Interdiscip. Appl. Math. 8, Springer-Verlag, New York, 1998. 
[13] L.D. Landau And E.M. Lifschitz, Elasticity Theory, Pergamon Press, Oxford, UK, 1975.

[14] H. Le Dret, Problemes variationnels dans les multidomaines, Rech. Math. Appl. 19, Masson, Paris, 1991.

[15] J.-L. Lions, Perturbations singulières dans les problèmes aux limites et en controle optimal, SpringerVerlag, Berlin, 1973.

[16] P. Luchini, M. Lupo, And A. Pozzi, Unsteady Stokes flow in a distensible pipe, ZAMM Z. Angew. Math. Mech., 71 (1991), pp. 367-378.

[17] A. Mikelić And R. TAPIÉRO, Mathematical derivation of the power law describing polymer flow through a thin slab, M2AN Math. Model. Numer. Anal., 29 (1995), pp. 3-22.

[18] F. Murat And A. Sili, Problèmes monotones dans des cylindres de faible diamètre, C. R. Acad. Sci. Paris Sér. I Math., 319 (1994), pp. 567-572.

[19] S.A. NAzArov, Asymptotic solution of the Navier-Stokes problem on the flow of a thin layer of fluid, Siberian Math J., 31 (1990), pp. 296-307.

[20] M.S. Olufsen, C.S. Peskin, W.Y. Kim, E.M. Pedersen, A. Nadim, and J. Larsen, Numerical simulation and experimental validation of blood flow in arteries with structured-tree outflow conditions, Annals of Biomedical Engineering, 28 (2000), pp. 1281-1299.

[21] M.S. Olufsen, A structured tree outflow condition for blood flow in the larger systemic arteries, Amer. J. Physiology, 276 (1999), pp. 257-268.

[22] K. Perktold AND G. RAPPITSCh, Mathematical modeling of local arterial flow and vessel mechanics, in Computational Methods for Fluid-Structure Interaction, Pitman Res. Notes Math. Ser. 306, J. Crolet and R. Ohayon, eds., Longman, Harlow, UK, 1994, pp. 230-245.

[23] A. Quarteroni, M. Tuveri, And A. Veneziani, Computational vascular fluid dynamics: Problems, models and methods. Survey article, Comput. Vis. Sci., 2 (2000), pp. 163-197.

[24] I. Tolstoy, Ed., Acoustics, Elasticity, and Thermodynamics of Porous Media. Twenty-One Papers by M.A. Biot, Acoustical Society of America, New York, 1992. 